\title{
An Evaluation of Euler Fluxes for Hypersonic Flow Computations
}

\author{
Keiichi Kitamura ${ }^{*}$ and Philip Roe ${ }^{\dagger}$ \\ Department of Aerospace Engineering, University of Michigan, Ann Arbor, MI 48109-2140, USA \\ and \\ Farzad Ismail ${ }^{\sharp}$ \\ IIHR-Hydroscience \& Engineering, University of Iowa, Iowa City, IA 52242-1585, USA
}

\begin{abstract}
Shock-capturing finite-volume schemes often give rise to anomalous results in hypersonic flow. We present here a wide-ranging survey of numerical experiments from eleven different flux functions in one- and two-dimensional contexts. Included is a recently-developed function that guarantees entropy stability.
\end{abstract}

\section{Nomenclature}

$e \quad=$ internal energy

$e_{t} \quad=$ total energy, $e+(1 / 2)\left(u^{2}+v^{2}\right)$

$p \quad=$ pressure, $(\gamma-1) \rho e$

$i, j \quad=$ cell indices

$u, v=$ velocity components

$x, y=$ Cartesian coordinates

$\gamma \quad=$ specific heat ratio, 1.4

$H \quad=$ total enthalpy, $e_{t}+p / \rho$

$M=$ Mach number

$S \quad=$ entropy, $\ln p-\gamma \ln \rho$

$\alpha=$ coefficient in new scheme

$\delta \quad=$ grid stretch parameter, $0,1 / 8, \ldots, 1$

$\varepsilon \quad=$ shock position parameter, $0.0,0.1, \ldots, 0.9$

$\rho \quad=$ density

Subscripts

$0=$ freestream value

$L \quad=$ left (pre) state of the shock

$M \quad=$ intermediate state of the shock

$R \quad=$ right (post) state of the shock

\section{Introduction}

$\mathrm{T}$ HE computation of hypersonic flows has proved surprisingly troublesome on account of anomalies which afflict many of the common schemes used for shock-capturing. Several schemes have been published with claims that they do not suffer from this effect. However, it is difficult to establish such claims theoretically, because we still lack an accepted explanation for the breakdowns. It is also difficult to establish them experimentally, because the phenomena depend on mesh geometry, mesh size, flow Mach number, and specific heat ratio. In this paper we pursue an experimental comparison. We will pay particular attention to those schemes known to fail ${ }^{1,2}$ and

\footnotetext{
*Ph.D. Student, keiichik@umich.edu; Exchange Student from Nagoya University, JAPAN, kitamura@fluid.nuae.nagoya-u.ac.jp, and AIAA Student Member

$\dagger$ Professor and AIAA Fellow

* Post Doctoral Associate and AIAA Member
} 
to schemes specifically claimed to avoid the phenomenon. ${ }^{3-7}$ Also, we will focus on a recently-published method ${ }^{8,9}$ that is an entropy-consistent development of the Roe scheme.

The carbuncle phenomenon appears to be very complex, and we feel convinced that there is no single cause, nor is there any single cure. Theoretical discussion is hampered by the fact that the carbuncle is a real physical solution, and so cannot be excluded by the application of any simple physical principle. Nevertheless, in studying it, we take a viewpoint that is partly physical and partly numerical.

We have organized our investigation around the hypothesis that part of the mechanism for generating the carbuncle is one-dimensional, and part is multidimensional. Thus, we begin by analyzing the apparently trivial problem of a steady shock in one dimension. It is surprising that this problem presents any difficulty, but as first pointed out by Barth ${ }^{10}$ the issue is that a stable solution must exist with the shock at any arbitrary location on the mesh, and this property is not enjoyed by several popular flux functions (Godunov, Roe, two-shock). This defect may be related to the fact that these schemes also fail to satisfy the Second Law of Thermodynamics in a strong sense. The failure occurs within the internal structure of a shock, and is distinct from the failure of the Roe scheme, for example, to eliminate rarefaction shocks. In our investigations, we have included a modified Roe scheme that does enforce the Second Law locally. It greatly improves the situation in 1D, although there is a tunable parameter involved. It turns out to be more effective than the common "entropy fix" due to Harten. ${ }^{11}$

Our next experiments are what we refer to as $11 / 2$ dimensional, in which we simply stack identical onedimensional problems on top of each other to form a two-dimension mesh of squares. As might be expected, schemes that fail the one-dimensional test also fail this test. However, even the schemes that pass the first test fail this one. The shock and the flow behind it develop two-dimensional modes. Thus, our initial expectation that the two-dimensional instability is driven by the one-dimensional instability is false. Several authors who have proposed fluxes that are intended to cure the carbuncle have recommended an additional dissipation having a multidimensional character. Such dissipation has no effect on the one-dimensional modes and is therefore ineffective unless the one-dimensional test is passed.

Finally, we consider the flow past a circular cylinder, using a grid provided by Dr Jeffery White of NASA Langley, in which one mesh line (set of cell interfaces) coincides with the shock obtained from a shock-fitting code. Naively, one might expect that mesh alignment of this kind would make it easy to capture the shock, but of course the $11 / 2$-dimensional test refutes this expectation. We made a series of tests in which the mesh was progressively dilated until, near the shock, the mesh lines were displaced by precisely one cell width. Thus, the shock took up all possible locations relative to the grid just as in the previous experiments. Disappointingly, the previous experiments did not invariably predict the outcome of this one, although there were correlations. It is possible that some third effect needs to be taken into account, but there are still many combinations of the two current approaches needing to be explored.

\section{Governing Equations}

The governing equations are two-dimensional compressible Euler equations as follows:

$$
\begin{aligned}
& \frac{\partial \mathbf{u}}{\partial t}+\frac{\partial \mathbf{F}}{\partial x}+\frac{\partial \mathbf{G}}{\partial y}=0 \\
& \mathbf{u}=\left[\begin{array}{c}
\rho \\
\rho u \\
\rho v \\
\rho e_{t}
\end{array}\right], \quad \mathbf{F}=\left[\begin{array}{c}
\rho u \\
\rho u^{2}+p \\
\rho u v \\
\rho u H
\end{array}\right], \quad \mathbf{G}=\left[\begin{array}{c}
\rho v \\
\rho v u \\
\rho v^{2}+p \\
\rho v H
\end{array}\right]
\end{aligned}
$$

where $\rho$ is density, $u$ and $v$ velocity components in Cartesian coordinates, $p$ pressure, $e_{t}$ total energy, $H$ total enthalpy

$\left(H=e_{t}+p / \rho\right)$. The calorically perfect gas model is assumed for air with the specific heat ratio $\gamma=1.4$. These equations are solved by the finite volume method (FVM). Both the spatial and time accuracies are $1^{\text {st }}$-order if not mentioned otherwise.

\section{Entropy-Stable and Entropy-Consistent Schemes}

The new entropy-stable scheme is described in Refs. 8 and 9. Basically, the standard formula

$$
\mathbf{F}^{*}=\mathbf{F}-\frac{1}{2} \mathbf{R}|\hat{\Lambda}| \mathbf{L} \Delta \mathbf{u}
$$

is replaced by 


$$
\mathbf{F}^{*}=\mathbf{F}_{c}-\frac{1}{2} \mathbf{R}|\hat{\mathbf{\Lambda}} \hat{\mathbf{S}}| \mathbf{R}^{\mathrm{T}} \Delta \mathbf{v}
$$

where $\mathbf{F}_{C}$ is a special averaging $\left(^{\wedge}\right)$ of the left and right states which conserves entropy,

$$
\mathbf{F}_{c}=\left[\hat{\rho} \hat{u}, \hat{\rho} \hat{u}^{2}+\hat{p}_{1}, \hat{\rho} \hat{u} \hat{v}, \hat{\rho} \hat{u} \hat{H}\right]^{\mathrm{T}}
$$

$\mathbf{S}$ is a scaling factor,

$$
\mathbf{S}=\operatorname{diag}\left(\frac{\hat{\rho}}{2 \gamma}, \frac{(\gamma-1) \hat{\rho}}{\gamma}, \hat{p}_{1}, \frac{\hat{\rho}}{2 \gamma}\right)
$$

and the dissipation term is not driven by the jump $\Delta \mathbf{u}$ in the conserved variables, but by the jump $\Delta \mathbf{v}$ in the entropy variables

$$
\Delta \mathbf{v}=\left[\Delta\left(\frac{\gamma-S}{\gamma-1}-\frac{\rho\left(u^{2}+v^{2}\right)}{2 p}\right), \Delta\left(\frac{\rho u}{p}\right), \Delta\left(\frac{\rho v}{p}\right),-\Delta\left(\frac{\rho}{p}\right)\right]^{\mathbf{T}}
$$

The diagonal matrix of eigenvalues is replaced by $|\hat{\mathbf{\Lambda}}| \rightarrow|\hat{\mathbf{\Lambda}}|+\alpha|\Delta \mathbf{\Lambda}|$ where the additional term $\alpha|\Delta \mathbf{\Lambda}|$ is introduced to ensure that the entropy produced by a shock is of third order as it should be. The coefficient alpha is not rigorously derived. For a weak shock sharply resolved it should be $1 / 6$. For stronger or less well-resolved shocks it needs to be larger and we are presently engaged in trying to make this more precise. This scheme is called entropy-consistent scheme and referred to as the new scheme in this paper. Because the analysis on which it is based is only semi-discrete we have employed a small Courant number when applying this scheme (see Table 1).

Table 1. CFL number chosen for each freestream Mach number $M_{0}$.

\begin{tabular}{c|ccc}
\hline$M_{0}$ & $1.5-6.0$ & 10 & 20 \\
\hline CFL & 0.5 & 0.2 & 0.1 \\
\hline
\end{tabular}

\section{Numerical Experiments}

\section{A. One Dimensional Problem: Steady Normal Shock}

Analytically a shock wave is regarded as a thin jump discontinuity, but a captured shock has internal structure. However, it is hard to establish what this internal structure should be. ${ }^{10,12,13}$ For example, the Godunov and Roe schemes produce an intermediate state that lies on the Hugoniot curve joining $\mathbf{u}_{R}$ to $\mathbf{u}_{L}$, but such a state does not preserve mass flux inside the shock. On the other hand, at least one intermediate state is needed to allow representation of a shock that is not located at a mesh interface. Therefore we prescribe initial conditions that include an intermediate state and boundary conditions that force the shock to remain in its initial position. The grid comprises 50 equally spaced cells as in Fig. 1 with initial

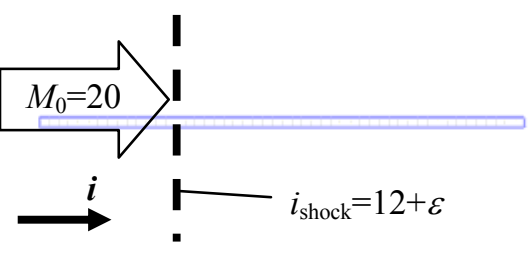

Figure 1. Computational Grid and Conditions for 1D Steady Shock Test Problem. conditions for left $(L: i \leq 12)$ and right $(R: i \geq 14)$ :

$$
\mathbf{u}_{L}=\left[\begin{array}{c}
1 \\
1 \\
0 \\
\frac{1}{\gamma(\gamma-1) M_{0}^{2}}+\frac{1}{2}
\end{array}\right], \quad \mathbf{u}_{R}=\left[\begin{array}{c}
f\left(M_{0}\right) \\
1 \\
0 \\
\frac{g\left(M_{0}\right)}{\gamma(\gamma-1) M_{0}^{2}}+\frac{1}{2 f\left(M_{0}\right)}
\end{array}\right]
$$

where

$$
f\left(M_{0}\right)=\left(\frac{2}{(\gamma+1) M_{0}^{2}}+\frac{\gamma-1}{\gamma+1}\right)^{-1}, \quad g\left(M_{0}\right)=\frac{2 \gamma M_{0}^{2}}{\gamma+1}-\frac{\gamma-1}{\gamma+1}
$$

following the Rankine-Hugoniot conditions across the normal shock. The internal shock conditions for $i=13$ (:M) are i) the density is given as

$$
\rho_{M}=\varepsilon \rho_{L}+(1-\varepsilon) \rho_{R}
$$


where the shock position parameter $\varepsilon=0.0,0.1, \ldots, 0.9$, and ii) the other variables are calculated based on $\rho_{M}$ so that all variables lie on the Hugoniot curve, connected to $\mathbf{u}_{\mathrm{L}}$ and $\mathbf{u}_{\mathrm{R}}$ as in Ref. 13. ${ }^{\S}$ The freestream Mach number is chosen in the range $1.5<M_{0}<20$. If a scheme is always stable for all those values of $\varepsilon$ and $M_{0}$, the scheme can be labeled as '1D stable.'

At the outflow boundary we prescribe the mass flux at the ghost cell $\left(i=i_{\max }+1\right)$;

$$
(\rho u)_{i \max +1, j}=(\rho u)_{0}=1
$$

in order for the mass in the whole computational domain to remain constant and for the shock to be fixed at the same position; meanwhile, other values are simply extrapolated, e.g., $\rho_{i \max +1, j}=\rho_{i \max , j}$. The inflow boundary has the freestream values.

Then, the computations are conducted until when (time steps) $\times(\mathrm{CFL})$ reached 20,000 (e.g., 100,000 steps with $\mathrm{CFL}=0.2$ ), and the CFL number is chosen depending on $M_{0}$ as in Table 1 , based on stability limit of the new scheme $^{8}$. In order to see how well upwind schemes preserve the initial shock position, we compare results of the new scheme with those of widely-used or recently proposed schemes, such as Roe's (original) scheme ${ }^{2}$ which is a linear approximate Riemann solver, Roe scheme with Harten's entropy fix (E-Fix) ${ }^{11}$ which removes 'expansion shock,' AUSM ${ }^{+4}{ }^{4} \mathrm{AUSM}^{+}$-up, ${ }^{5}$ AUSMPW ${ }^{7}$ and RoeM2 ${ }^{3}$ schemes that preserve total enthalpy $H$ in steady flow instead of energy, and HLLE $^{6}$ which is widely believed to be a Carbuncle-free but notoriously dissipative scheme. We do not show results for the exact (Godunov) Riemann solver, ${ }^{1}$ because its behavior is almost identical to that of the unmodified Roe scheme.

(a) New $(\alpha=0.2)$

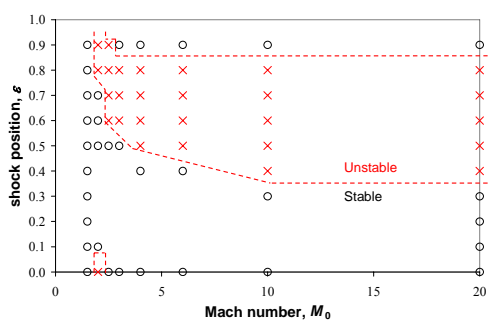

(d) Roe (E-Fix)

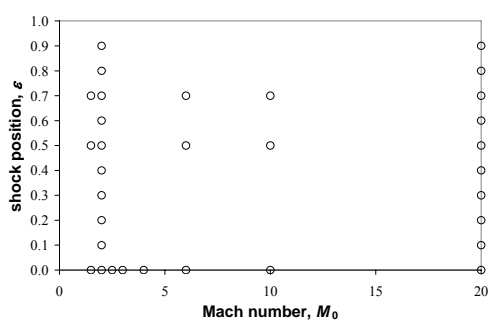

(g) AUSMPW+

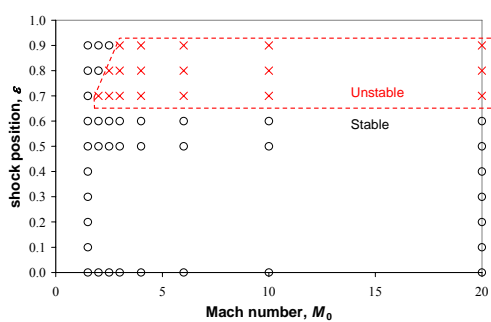

(b) $\operatorname{New}(\alpha=0.8)$

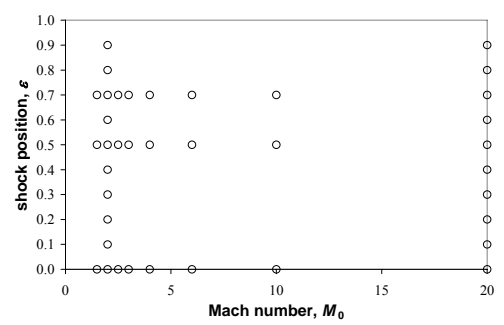

(e) AUSM+

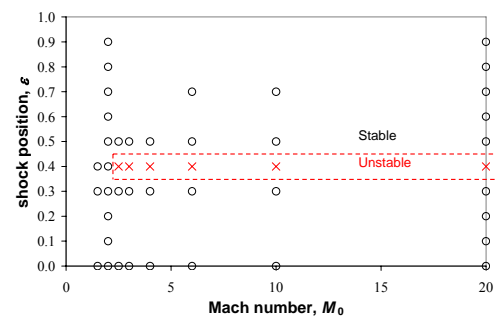

(h) RoeM2

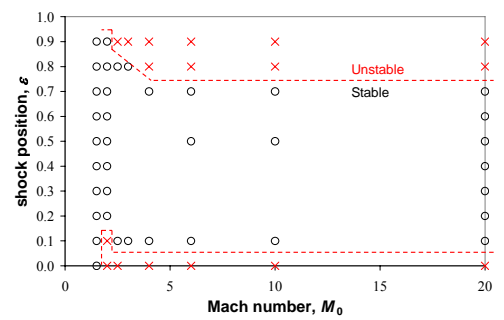

(c) Roe

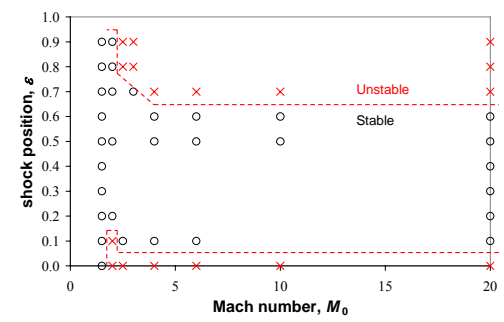

(f) $\mathrm{AUSM}^{+}$-up

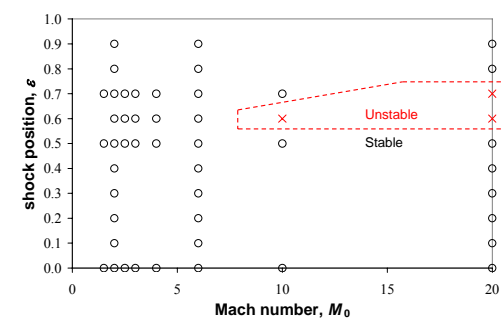

(i) HLLE

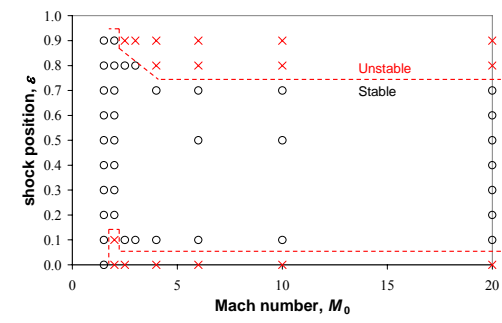

Figure 2. One-Dimensional Stability Limits for Upwind Schemes (Freestream Mach Number 1.5 $<M_{0}<20$, Shock Position $\varepsilon=0.0,0.1, \ldots, 0.9$ ).

\footnotetext{
$\S$ Though there are some alternative ways to determine internal shock states, e.g., all the primitive variables are $\mathbf{u}_{\mathrm{M}}=\varepsilon \mathbf{u}_{\mathrm{L}}+(1-\varepsilon) \mathbf{u}_{\mathrm{R}}$, we confirmed that these have minor effects on calculated flowfields.
} 
The behavior of the various schemes is summarized in Fig. 2. If the computation converged (L2-norm of density dropped at least three orders of magnitude) with the initial shock position, it is stable (labeled as circle: $\mathrm{O}$ ). If, on the other hand, the computation did not converge or the shock moved to either boundary and disappeared, the computation is unstable (cross: $\times$ ).

As can be seen, the new scheme with a large coefficient $(\alpha=0.8)$ and Roe scheme with E-Fix are 1D stable in all cases, whereas others are not. The other schemes are stable only for low Mach numbers $\left(M_{0}<10\right.$ for AUSM+-up, $M_{0}<2.5$ for AUSM+, and $M_{0}<1.5$ for the others), or for particular shock positions depending on the schemes. Thus, most schemes claimed as 'Carbuncle-free' are actually not stable in a 1D calculation. This aspect of the carbuncle has been largely ignored by researchers, but our contention is that one-dimensional stability is a necessary (although not sufficient) condition for curing the carbuncle. In the following subsections, we will extend this discussion to two-dimensional problems.

\section{B. 1 1/2 Dimensional Problem: Steady Normal Shock}

Next we solved a steady shock which is initially aligned in one direction

(a) New ( $\alpha=0.2), \varepsilon=0.0$

Stable

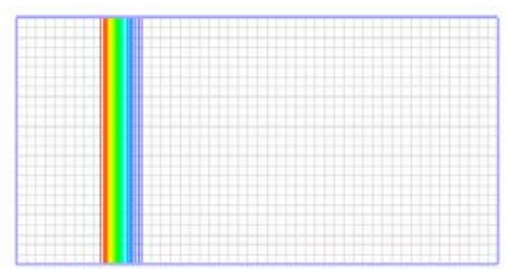

(d) Roe (E-Fix), $\varepsilon=0.0$ Unstable (Disappeared)

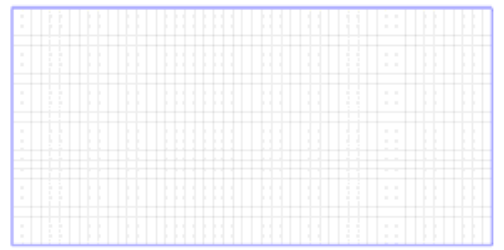

(g) AUSMPW,$+ \varepsilon=0.5$ Stable

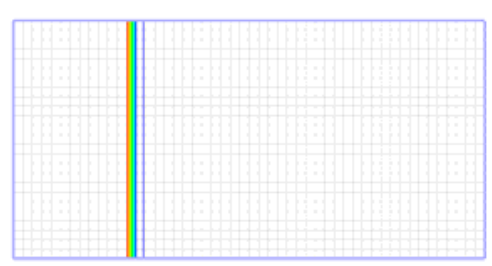

(j) AUSMPW,$+ \varepsilon=0.5$

(without multidimensional term)

Unstable (Asymmetric)

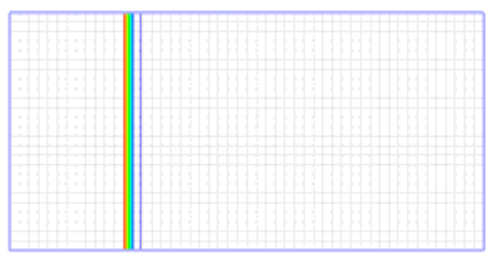

(b) New ( $\alpha=0.8$ ), $\varepsilon=0.0$ Unstable (Disappeared)

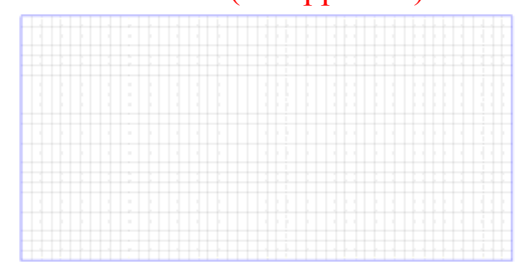

(e) AUSM,$+ \varepsilon=0.0$ Unstable (Stage 1)

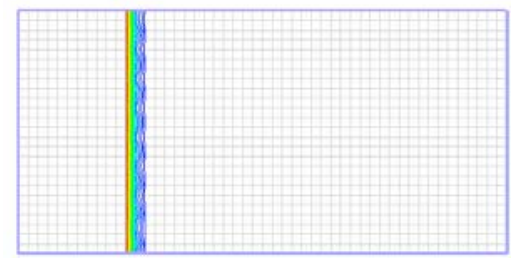

(h) RoeM2, $\varepsilon=0.5$

Stable

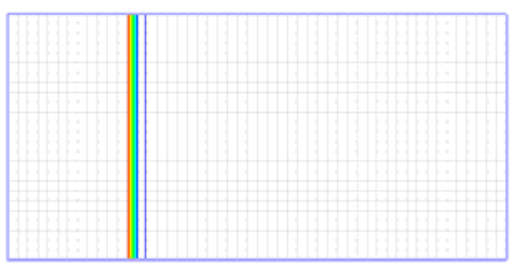

(k) RoeM2, $\varepsilon=0.5$

(without multidimensional term) Unstable (Asymmetric)

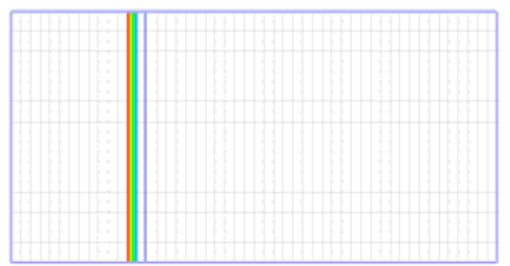

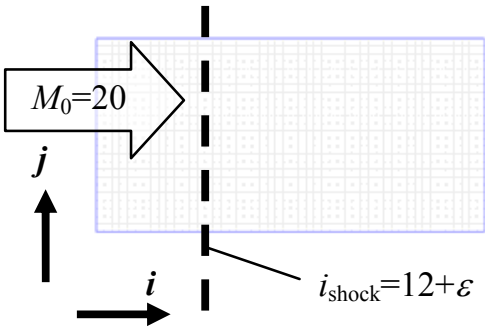

Figure 3. Computational Grid and Conditions for 1 1/2 D Steady Shock Test Problem.

(c) Roe, $\varepsilon=0.5$

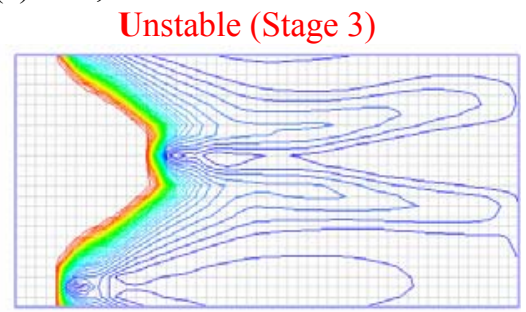

(f) $\mathrm{AUSM}^{+}$-up, $\varepsilon=0.0$

Unstable (Stage 2)

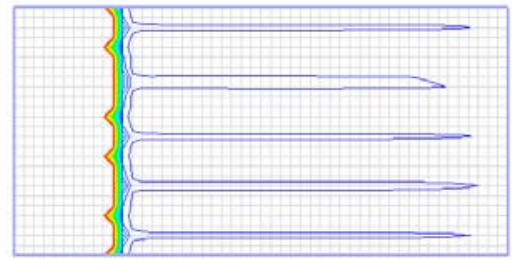

(i) HLLE, $\varepsilon=0.5$

Stable

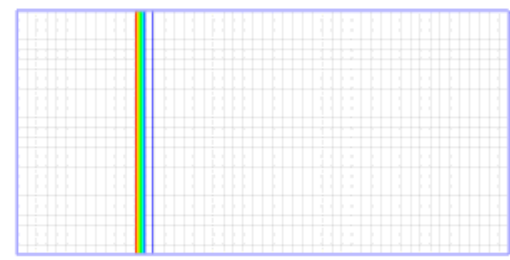

(1) $\mathrm{AUSM}+, \varepsilon=0.0,50,000$ steps

(Stable)

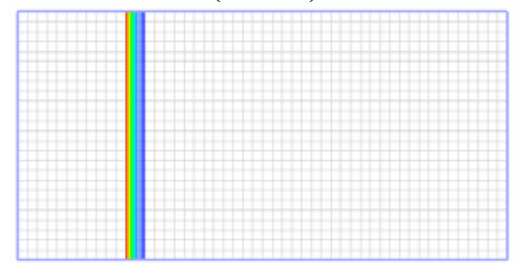

Figure 4. Mach Number Contours at 200,000 time steps (except for (l)) for 1 1/2 Dimensional Steady Shock (Freestream Mach Number $M_{0}=20$, Shock Position $\varepsilon$ is One-Dimensionally Stable). 
in a two-dimensional (2D) field (Fig. 3). We expect that such a computed flowfield should behave in a onedimensional (1D) manner, and thus, we call this problem " $11 / 2$ dimensional $(11 / 2 \mathrm{D})$ problem." This is a simplified carbuncle problem which was developed first by Quirk ${ }^{14}$ and modified by Dumbser et al. ${ }^{15}$ but we employ a downstream boundary condition, similar to that employed in $1 \mathrm{D}$, that prevents an unstable shock from simply relocating to a stable position. Also we used a grid that is extended farther downstream from the shock. We found that this made the development of unstable solutions more likely. In particular, we employ a grid having $50 \times 25$ cells spaced evenly without any perturbation. The freestream Mach number is $M_{0}=20$, the periodical condition is imposed for the boundaries of $j$-direction, and the other initial conditions and boundary conditions are the same as the 1D tests. The computations were conducted for 200,000 steps with $\mathrm{CFL}=0.1$. If a scheme is stable for all the shock positions $\varepsilon$, the scheme can be labeled as ' $11 / 2 \mathrm{D}$ stable.'

\section{- Shock Locations that are Stable in 1D}

A selection of our computations is presented in Figs 4 and 5. In Fig. 4, Mach number contours at 200,000 time steps are shown, and Fig. 5 shows corresponding residual (L2-norm of density) histories of these calculations. We show only cases that were stable in $1 \mathrm{D}$. No case that was unstable in $1 \mathrm{D}$ proved to be stable in $11 / 2 \mathrm{D}$ as will be shown later (Figs. 7 and 8), and therefore we claim that 1D stability is necessary for $11 / 2 \mathrm{D}$ stability. The tests reported in this section determine whether it is also sufficient, and if not, how the $11 / 2 \mathrm{D}$ instability develops. The instabilities that appeared sometimes took the form of local oscillations confined to the shock (Stage 1), streaks of vorticity streaming behind the shock (Stage 2 ) or total breakdown (Stage 3). ${ }^{8,16}$ In the last case, the density behind the shock is no longer that behind a normal shock, and even under our new boundary condition the shock is free to

(a) New $(\alpha=0.2), \varepsilon=0.0$

Stable

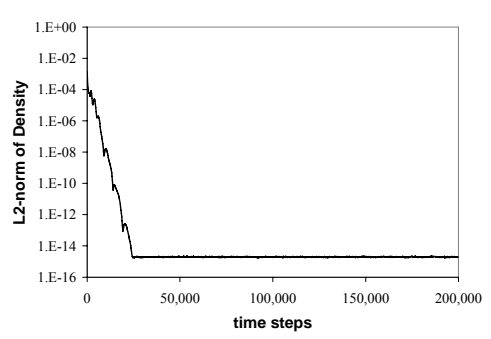

(d) Roe (E-Fix), $\varepsilon=0.0$

Unstable (Disappeared)

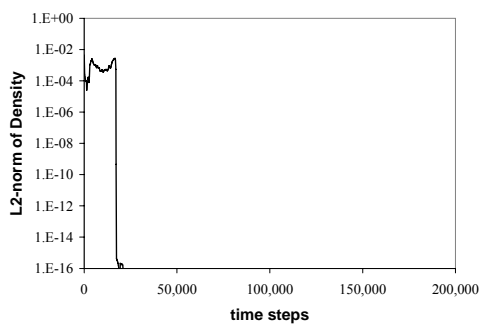

(g) AUSMPW,$+ \varepsilon=0.0$

Stable/ Unstable (Asymmetric)

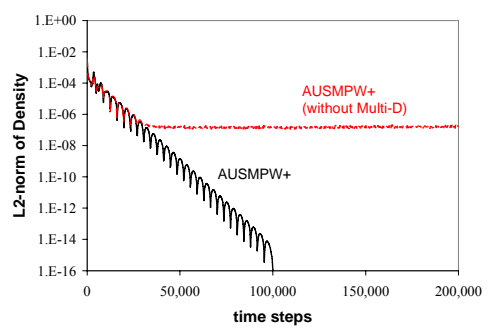

(b) New ( $\alpha=0.8), \varepsilon=0.0$ Unstable (Disappeared)

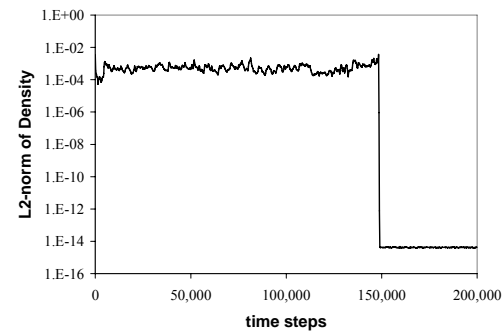

(e) $\mathrm{AUSM}+, \varepsilon=0.0$

Unstable (Stage 1)

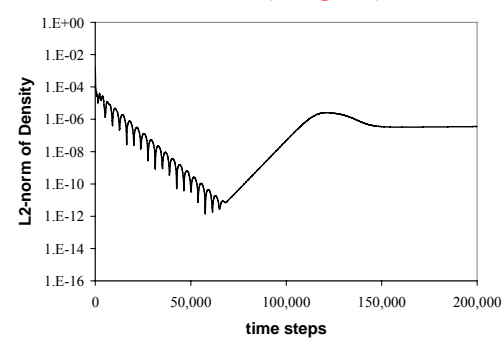

(h) RoeM2, $\varepsilon=0.5$

Stable/ Unstable (Asymmetric)

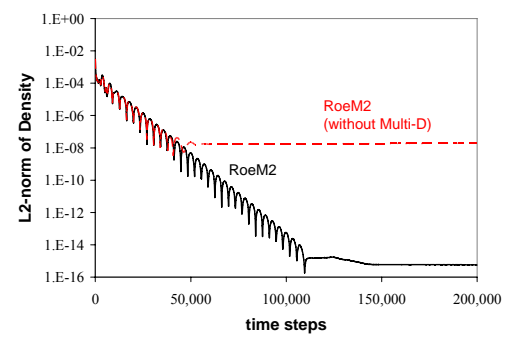

(c) Roe, $\varepsilon=0.5$

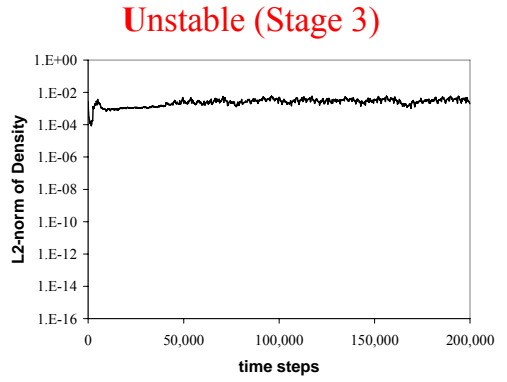

(f) $\mathrm{AUSM}^{+}$-up, $\varepsilon=0.0$

Unstable (Stage 2)

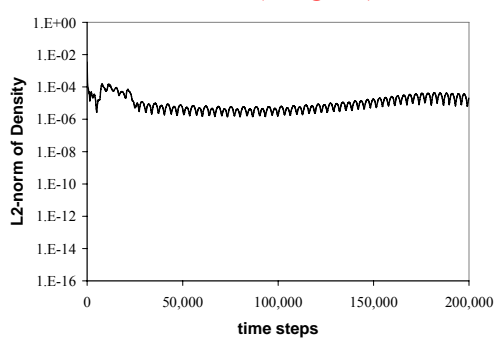

(i) HLLE, $\varepsilon=0.5$

Stable

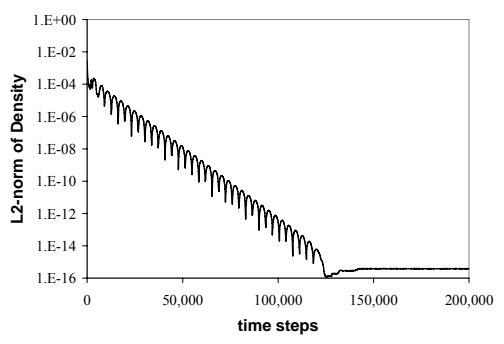

Figure 5. Residual Histories for 1 1/2 Dimensional Steady Shock (Freestream Mach Number $M_{0}=\mathbf{2 0}$, Shock Position $\varepsilon$ is One-Dimensionally Stable). 
move, and may disappear off the grid. The following features of these results are noteworthy:

- The only two schemes that were universally stable in $1 \mathrm{D}$ (the new scheme with $\alpha=0.8$ and Roe's scheme with Harten's E-fix) both failed this test (Figs. 4b, 4d). Moreover, many of the schemes that were stable in 1D for some particular combination of $\left(M_{0}, \varepsilon\right)$ are unstable here. Hence 1D stability is not sufficient for 1 1/2D stability.

- $\mathrm{AUSM}^{+}$solution has a surprising feature: after the residual converged to $O\left(10^{-11}\right)$ with an apparently stable solution (Fig. 41), the flowfield suddenly destabilized around 60,000 steps. After that, the residual grew exponentially, and dropped again (Fig. 5e). Eventually, the calculation reached to Stage 1 Carbuncle solution (200,000 steps, Fig. 4e). This scheme cannot be called '1 1/2D stable,' and further explanation will appear later.

- $\mathrm{AUSM}^{+}$-up scheme showed Stage 2 Carbuncle (Fig. 4f) and the residual stagnated around $O\left(10^{-5}\right)$ (Fig. 5f). This

(a) New ( $\alpha=0.2), \varepsilon=0.0$

Stable

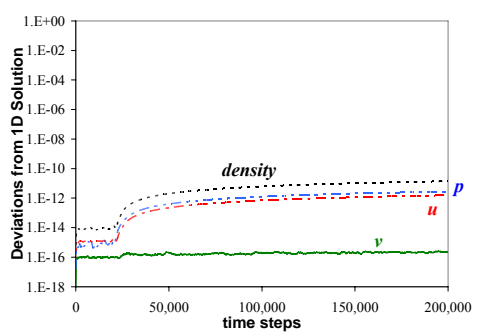

(d) Roe (E-Fix), $\varepsilon=0.0$

Unstable (Disappeared)

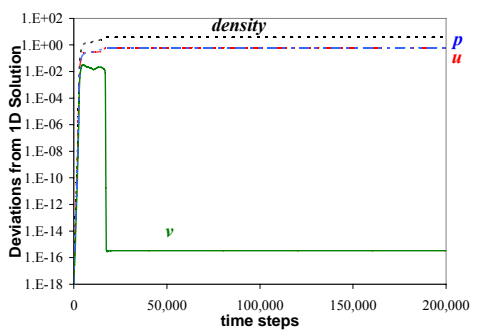

(g) AUSMPW,$+ \varepsilon=0.5$

Stable

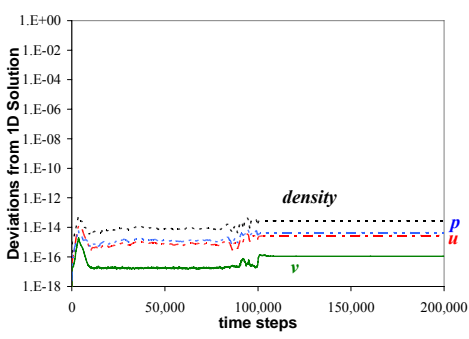

(j) AUSMPW,$+ \varepsilon=0.5$

(without multidimensional term)

Unstable (Asymmetric)

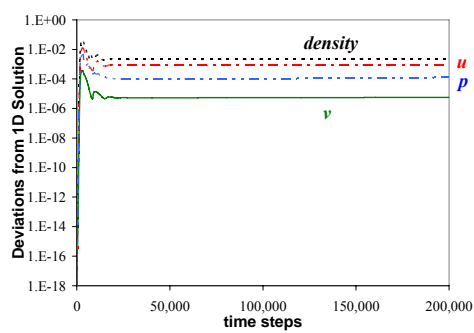

(b) New ( $\alpha=0.8$ ), $\varepsilon=0.0$

Unstable (Disappeared)

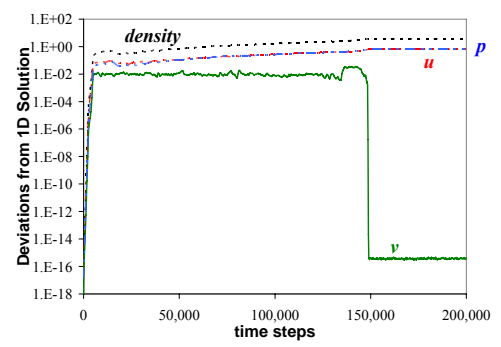

(e) $\mathrm{AUSM}+, \varepsilon=0.0$

Unstable (Stage 1)

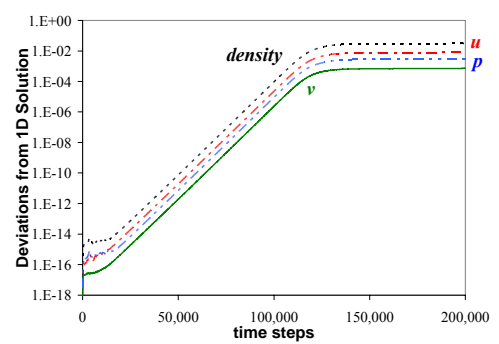

(h) RoeM2, $\varepsilon=0.5$

Stable

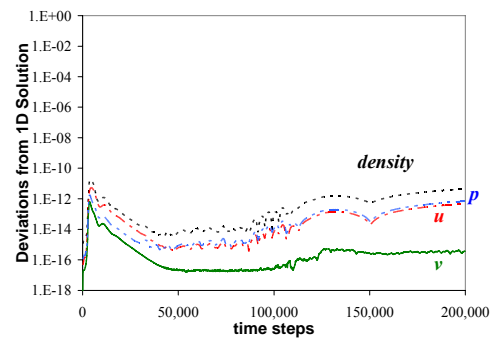

(k) RoeM2, $\varepsilon=0.5$

(without multidimensional term)

Unstable (Asymmetric)

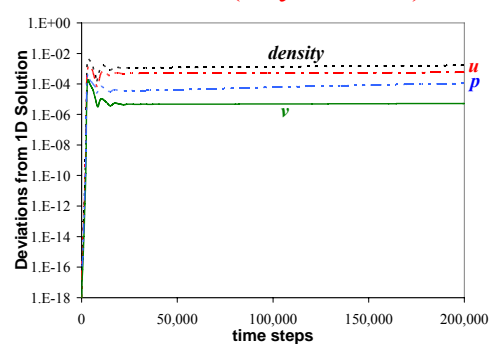

(c) Roe, $\varepsilon=0.5$

Unstable (Stage 3)

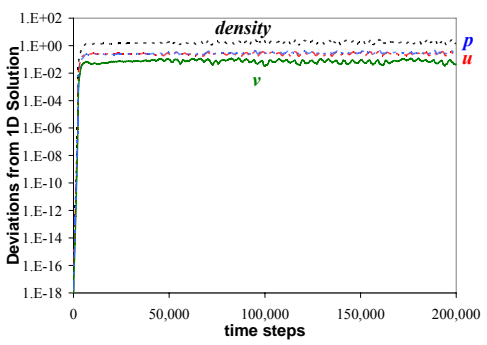

(f) $\mathrm{AUSM}^{+}$-up, $\varepsilon=0.0$

Unstable (Stage 2)

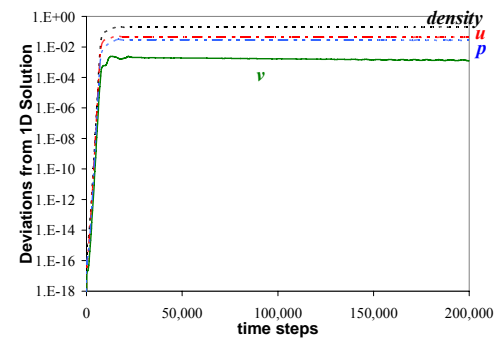

(i) HLLE, $\varepsilon=0.5$

Stable

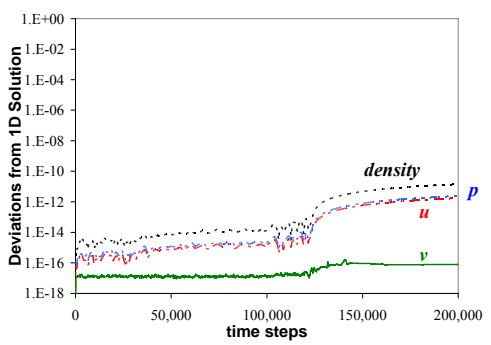

Figure 6. Histories of Deviations of Primitive Variables from 1D Solutions (Freestream Mach Number $M_{0}=\mathbf{2 0}$, Shock Position $\varepsilon$ is One-Dimensionally Stable).

7 of 21

American Institute of Aeronautics and Astronautics 
scheme, again, is not ' $11 / 2 \mathrm{D}$ stable.'

- Both AUSMPW+ and RoeM2 schemes have multidimensional terms and are claimed to be as 'Carbuncle-free.' With these terms, as can be expected, the results were stable whenever the 1D case was stable (Figs. 4g, 4h, 5g, 5h). However, when those multidimensional terms were eliminated, the solutions did not converge (Figs. 5g, 5h). Although it is not clearly seen from Figs. $4 \mathrm{j}$ and $4 \mathrm{k}$, these solutions are not symmetric (:differences of density between cells at different $j$-index with the same $i$-index downstream the shock were $O\left(10^{-3}\right)$, while this differences were smaller than $O\left(10^{-13}\right)$ for results labeled as 'Stable').

- HLLE scheme, the only scheme known as 'Carbuncle-free' among widely-used schemes (though it lacks resolution of a contact discontinuity or a boundary layer) showed a stable result, whenever the 1D case was stable.

In search of more insight, we measured how the unstable 11/2D solutions deviated from the stable 1D solutions, specifically we computed the L1 norm of the primitive variables $(\rho, u, v, p)$. Figure 6 shows time histories of the deviations from the $1 \mathrm{D}$ solutions. Noteworthy conclusions are:

- In all of the unstable cases except one, the deviation grew rapidly and immediately in all variables. The exception was AUSM+ whose growth was very gentle. This accounts for the apparently satisfactory stability of this scheme at early times.

- Even in those cases that we classified as stable, the solutions did not remain identical to the 1D solutions; there was very slow growth of a systematic deviation, and in cases (a), (h) and (i) one may speculate that even after 200,000 time steps there could be more to come.

- The only case that seems to be undeniably converged is AUSMPW+ (g). Both this scheme and RoeM2 (h) are equipped with multidimensional dissipation. If this dissipation is removed $(\mathrm{j}),(\mathrm{k})$, then both methods rapidly deviate from the 1D solutions, and the residual "hangs," even though the solutions look good in Figs. 4j and 4k.

- Shock Locations that are Unstable in 1D

In Figs. 7 and 8 we present results for cases that were unstable in 1D. All of these cases except one, including all

(a) New ( $\alpha=0.2), \varepsilon=0.5$

Unstable (Stage 3)

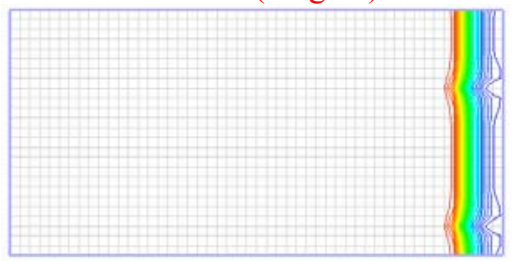

(d) $\mathrm{AUSM}^{+}$-up, $\varepsilon=0.7$

Unstable (Stage 2)

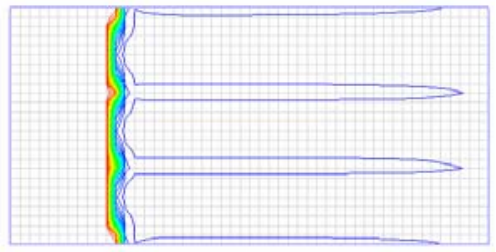

(g) HLLE, $\varepsilon=0.0$

Unstable (1D instability)

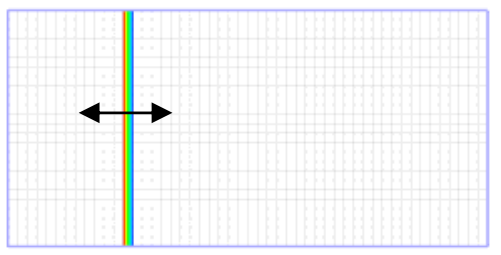

(b) Roe, $\varepsilon=0.0$

Unstable (Stage 3)

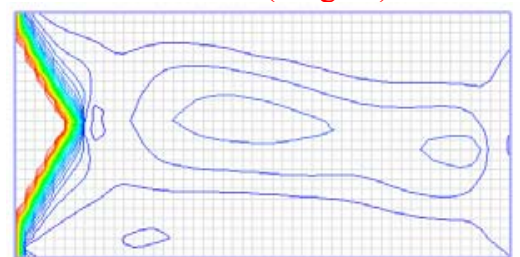

(e) AUSMPW,$+ \varepsilon=0.8$

Unstable (Stage 1)

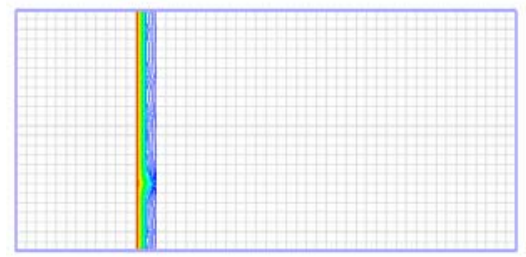

(h) AUSMPW+, $\varepsilon=0.8$

(without multidimensional term)

Unstable (Stage 1)

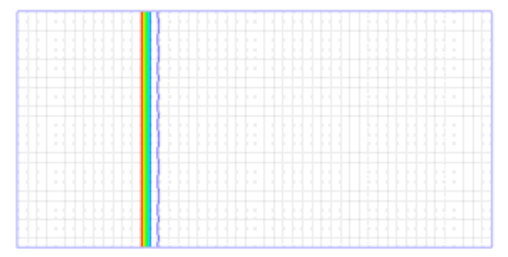

(c) AUSM,$+ \varepsilon=0.4$ Unstable (Stage 1)

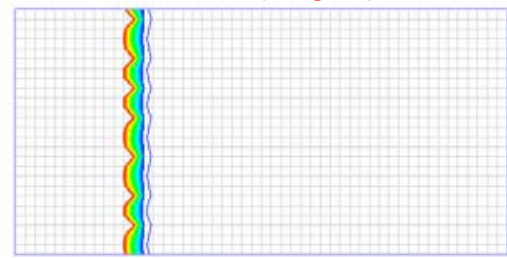

(f) RoeM2, $\varepsilon=0.0$

Unstable (1D instability)

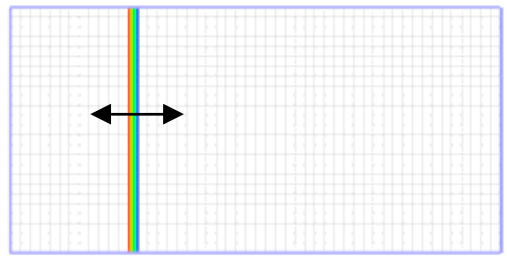

(i) RoeM2, $\varepsilon=0.0$

(without multidimensional term)

Stable

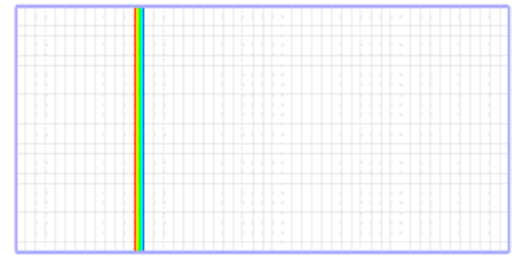

Figure 7. Mach Number Contours at 200,000 time steps for 1 1/2 Dimensional Steady Shock (Freestream Mach Number $M_{0}=20$, Shock Position $\varepsilon$ is One-Dimensionally Unstable). 
of the cases that we have no space to show, were unstable in $11 / 2 \mathrm{D}$. This includes even the cases (e) and (f) that feature multidimensional dissipation. It seems that in case (e) the dissipation is able to partly suppress the additional modes but still leaves the first stage of instability. In case (f) the additional modes are completely suppressed, but the 1D mode remains. In (g), the HLLE scheme, because of its inability to sustain contact discontinuities, has a built-in dissipation that also completely suppresses the additional modes, but leaves the $1 \mathrm{D}$ mode in place. Case (h) even without the multidimensional term, does not progress beyond the first stage, but are definitely unstable. Case (i) is the only exception. As shown before, however, this version of RoeM2 scheme failed this test for another choice of shock location. Thus, by eliminating multidimensional dissipation, the scheme just changed its favorite shock location.

We confirmed our expectation that if a scheme is unstable in $1 \mathrm{D}$, then it remains unstable in $11 / 2 \mathrm{D}$ even if a multidimensional dissipation is added. This suggests that the schemes of Sanders $e t a .^{21}$ and Ren ${ }^{22}$ would also be unstable in $11 / 2 \mathrm{D}$ for certain cases, although we have not confirmed this by experiment.

In summary, we have found '1D stable' schemes (new scheme $(\alpha=0.8)$ and Roe (E-Fix)) but no '1 1/2D stable' schemes. The following schemes are stable only under certain shock locations: New scheme $(\alpha=0.2)$, AUSMPW + , RoeM2 (with and without multidimensional dissipation), and HLLE.

\section{Two Dimensional Problem: Hypersonic Flow over Blunt Body with Shock-Aligned Grid}

Finally, we will go on to a fully two-dimensional (2D) problem. Figure 5 shows the computational grid and conditions in this case. The grid has $48 \times 120$ cells and has been designed so that a fitted bow shock lies on an $i=$ const.

(a) New ( $\alpha=0.2), \varepsilon=0.5$

Unstable (Stage 3)

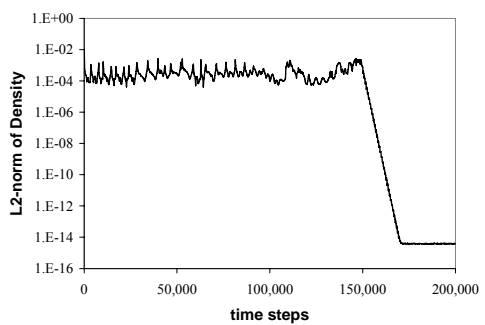

(d) $\mathrm{AUSM}^{+}$-up, $\varepsilon=0.7$

Unstable (Stage 2)

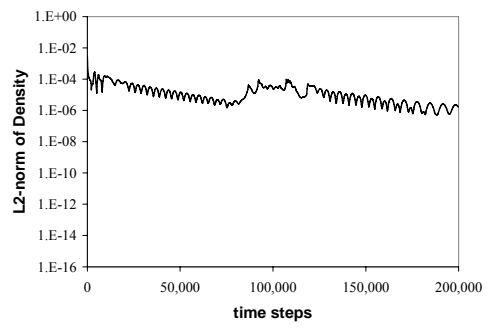

(g) HLLE, $\varepsilon=0.0$

Unstable (1D instability)

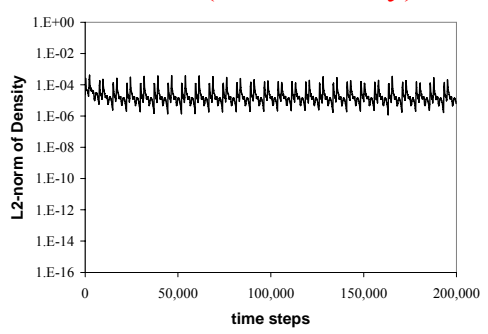

(b) Roe, $\varepsilon=0.0$

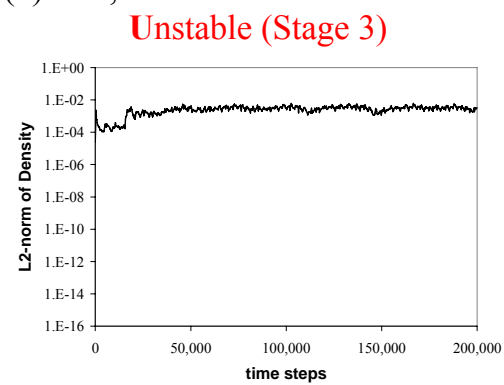

(e) AUSMPW,$+ \varepsilon=0.8$

Unstable (Stage 1)

/ Unstable (Stage 1)

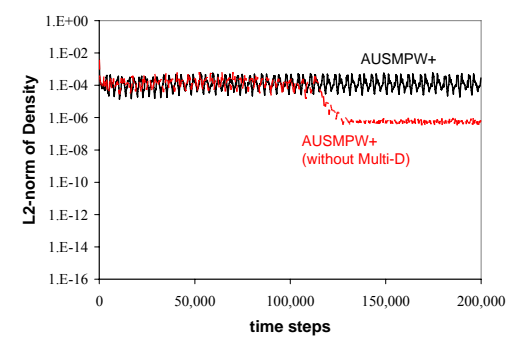

(c) AUSM+, $\varepsilon=0.4$

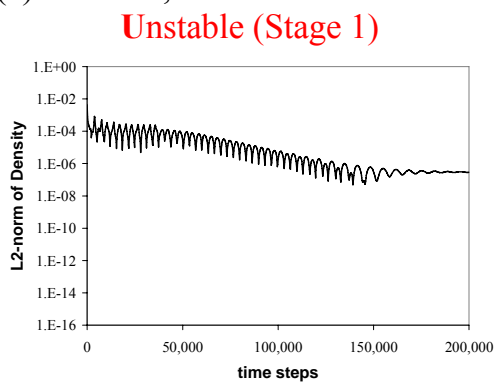

(f) RoeM2, $\varepsilon=0.0$

Unstable (1D instability)

/ Stable

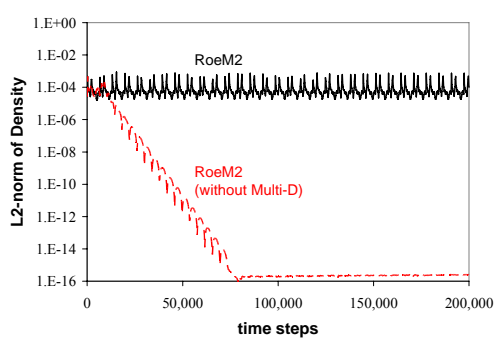

Figure 8. Residual Histories for 1 1/2 Dimensional Steady Shock

(Freestream Mach Number $M_{0}=20$, Shock Position $\varepsilon$ is One-Dimensionally Unstable).

9 of 21

American Institute of Aeronautics and Astronautics 
line for $M_{0}=6.0$ (Dr. Jeffery White et al. at NASA Langley, private communication). We checked that our version of this grid was perfectly symmetric. We then stretched this grid outward, controlling the motion of the $i=$ const. line that theoretically coincides with the shock position. We introduced a parameter $\delta$ such that if $\delta=0$ we recover the original grid but if $\delta=1$ the adjacent grid line moves to the theoretical shock location. We varied this parameter by intervals of $1 / 8$ so that, just as in the earlier tests, the shock took up a variety of locations relative to the grid. We expected that, if our results were stable for $\delta=0$ they were also stable for $\delta=1$. However, there were a few exceptions, perhaps because the captured shocks were not exactly aligned with the grid. As the parameter $\delta$ changes by unity so does the parameter $\varepsilon$, but they are not the same because the captured shocks will not be in exactly the same position as the fitted shock.

The specified condition at the inlet $(i=0)$ is just freestream Mach number of $M_{0}=6.0$ with reference density and pressure. The slip condition is applied at the wall $(i=i m a x+1)$, and the simple extrapolation is employed at the outlet $(j=0$ and $j m a x+1)$. Computations were conducted with $\mathrm{CFL}=0.5$ for 50,000 time steps unless the residual converged to machine zero. The spatial accuracy is $1^{\text {st }}$-order or $2^{\text {nd }}$-order by using MUSCL scheme ${ }^{19}$ with Van Albada's limiter. ${ }^{20}$

Two examples of computed flowfields are shown in Fig. 10. Compared here are results of $2^{\text {nd }}$-order Roe scheme with $\delta=0$ (no displacement) and $\delta=4 / 8$ (half cell displacement). The bow shock exactly lies on an $i=$ const. line and the solution converged successfully for $\delta=0$; however, for $\delta=4 / 8$ the shock seemed to look for comfortable position rather than settle down on particular $i=$ const. line and the solution did not converge.

In order to see whether the same or similar discussion holds to other fluxes, and to find some relation between these results with $1 \mathrm{D}$ or $11 / 2 \mathrm{D}$ results, we summarized various results in Table 2 for flux functions investigated in the preceding subsections. In this Table,

- 'S' denotes a case where the code converged steadily and exponentially toward a satisfactory solution.

- ' $U$ ' denotes a case where the code initially converged toward a satisfactory solution, but at some stage the residual "hung up" and the solution remained of poor quality. This case resembles Stages 1 and 2 of the carbuncle.

- 'A' denotes a case similar to $U$, but where the residual eventually

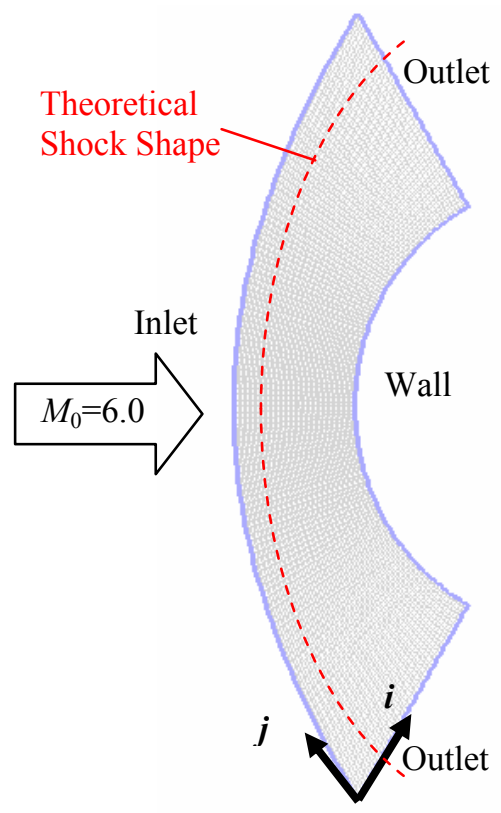

began to decrease again, with convergence to an unsatisfactory solution, usually asymmetric and in the form of a carbuncle.

(a) $\delta=0$

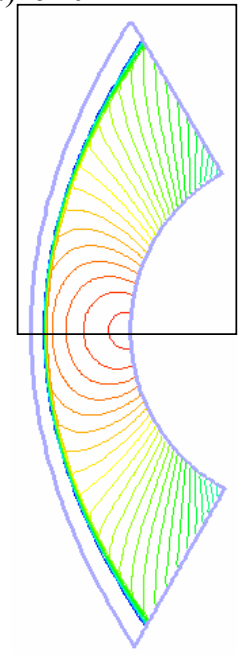

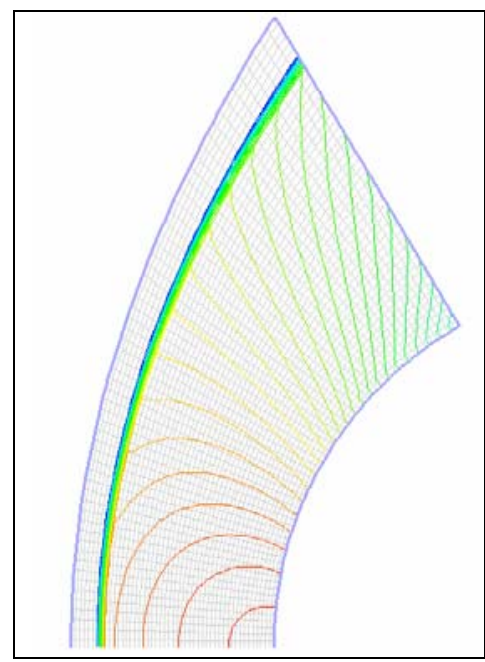

(b) $\delta=4 / 8$

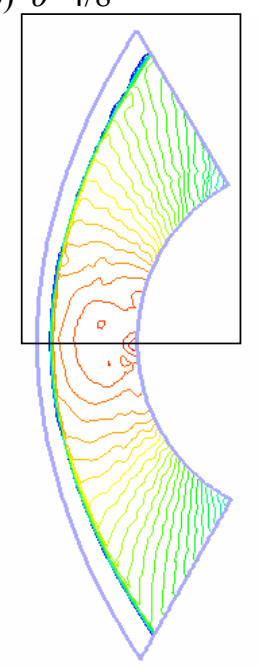

Figure 9. Computational Grid and Conditions for Blunt Body Problem.

Figure 10. Pressure Coefficient Contours with Grid around 2D Cylinder at 50,000 time steps (Roe, $2^{\text {nd }}-$ order).

10 of 21

American Institute of Aeronautics and Astronautics 
Table 2. Summary of Computed Results for 2D Cylinder with Various Schemes.

\begin{tabular}{|c|c|c|c|c|c|c|c|c|c|c|}
\hline Scheme & $\begin{array}{c}\text { Order of } \\
\text { Accuracy }\end{array}$ & $\delta=0$ & $1 / 8$ & $2 / 8$ & $3 / 8$ & $4 / 8$ & $5 / 8$ & $6 / 8$ & $7 / 8$ & 1 \\
\hline \multirow{2}{*}{$\begin{array}{c}\text { New } \\
(\alpha=0.2)\end{array}$} & $1^{\text {st }}$ & $\mathbf{U}$ & $\mathbf{U}$ & $\mathbf{A}$ & $\mathbf{S}$ & $\mathbf{S}$ & $\mathbf{S}$ & $\mathbf{S}$ & $\mathbf{A}$ & $\mathbf{U}$ \\
\hline & $2^{\text {nd }}$ & $\mathbf{U}$ & $\mathbf{U}$ & $\mathbf{U}$ & $\mathbf{U}$ & $\mathbf{S}$ & $\mathbf{S}$ & $\mathbf{S}$ & $\mathbf{S}$ & $\mathbf{U}$ \\
\hline \multirow{2}{*}{$\operatorname{New}(\alpha=0.8)$} & $1^{\text {st }}$ & $\mathbf{U}$ & $\mathbf{A}$ & $\mathbf{A}$ & $\mathbf{U}$ & $\mathbf{U}$ & $\mathbf{U}$ & $\mathbf{A}$ & $\mathbf{U}$ & $\mathbf{U}$ \\
\hline & $2^{\text {nd }}$ & $\mathbf{U}$ & $\mathbf{U}$ & $\mathbf{U}$ & $\mathbf{U}$ & $\mathbf{U}$ & $\mathbf{U}$ & $\mathbf{U}$ & $\mathbf{U}$ & $\mathbf{U}$ \\
\hline \multirow{2}{*}{ Roe } & $1^{\text {st }}$ & $\mathbf{A}$ & $\mathbf{A}$ & $\mathbf{A}$ & $\mathbf{U}$ & $\mathbf{U}$ & $\mathbf{A}$ & $\mathbf{A}$ & $\mathbf{A}$ & $\mathbf{U}$ \\
\hline & $2^{\text {nd }}$ & $\mathbf{S}$ & $\mathbf{U}$ & $\mathbf{U}$ & $\mathbf{U}$ & $\mathbf{U}$ & $\mathbf{U}$ & $\mathbf{S}$ & $\mathbf{S}$ & $\mathbf{S}$ \\
\hline \multirow{2}{*}{ Roe (E-Fix) } & $1^{\text {st }}$ & $\mathbf{A}$ & $\mathbf{A}$ & $\mathbf{A}$ & $\mathbf{A}$ & $\mathbf{A}$ & $\mathbf{A}$ & $\mathbf{A}$ & $\mathbf{A}$ & $\mathbf{A}$ \\
\hline & $2^{\text {nd }}$ & $\mathbf{S}$ & $\mathbf{S}$ & $\mathbf{U}$ & $\mathbf{U}$ & $\mathbf{U}$ & $\mathbf{U}$ & $\mathbf{S}$ & $\mathbf{S}$ & $\mathbf{S}$ \\
\hline \multirow{2}{*}{ AUSM+ } & $1^{\text {st }}$ & $\mathbf{S}$ & $\mathbf{S}$ & $\mathbf{S}$ & $\mathbf{S}$ & $\mathbf{S}$ & $\mathbf{S}$ & $\mathbf{S}$ & $\mathbf{S}$ & $\mathbf{S}$ \\
\hline & $2^{\text {nd }}$ & $\mathbf{S}$ & $\mathbf{U}$ & $\mathbf{S}$ & $\mathbf{S}$ & $\mathbf{S}$ & $\mathbf{S}$ & $\mathbf{S}$ & $\mathbf{S}$ & $\mathbf{S}$ \\
\hline \multirow{2}{*}{$\operatorname{AUSM}^{+}$-up } & $1^{\text {st }}$ & $\mathbf{U}$ & $\mathbf{U}$ & $\mathbf{U}$ & $\mathbf{S}$ & $\mathbf{S}$ & $\mathbf{S}$ & $\mathbf{S}$ & $\mathbf{A}$ & $\mathbf{U}$ \\
\hline & $2^{\text {nd }}$ & $\mathbf{S}$ & $\mathbf{S}$ & $\mathbf{U}$ & $\mathbf{U}$ & $\mathbf{S}$ & $\mathbf{U}$ & $\mathbf{U}$ & $\mathbf{U}$ & $\mathbf{U}$ \\
\hline \multirow{2}{*}{$\begin{array}{c}\text { AUSMPW+ } \\
\text { (with multi-D) }\end{array}$} & $1^{\text {st }}$ & $\mathbf{S}$ & $\mathbf{S}$ & $\mathbf{S}$ & $\mathbf{S}$ & $\mathbf{S}$ & $\mathbf{S}$ & $\mathbf{U}$ & $\mathbf{S}$ & $\mathbf{S}$ \\
\hline & $2^{\text {nd }}$ & $\mathbf{S}$ & $\mathbf{S}$ & $\mathbf{S}$ & $\mathbf{U}$ & $\mathbf{U}$ & $\mathbf{S}$ & $\mathbf{S}$ & $\mathbf{S}$ & $\mathbf{S}$ \\
\hline \multirow{2}{*}{$\begin{array}{c}\text { RoeM2 } \\
\text { (with multi-D) }\end{array}$} & $1^{\text {st }}$ & $\mathbf{S}$ & $\mathbf{S}$ & $\mathbf{S}$ & $\mathbf{A}$ & $\mathbf{U}$ & $\mathbf{S}$ & $\mathbf{S}$ & $\mathbf{S}$ & $\mathbf{S}$ \\
\hline & $2^{\text {nd }}$ & $\mathbf{S}$ & $\mathbf{S}$ & $\mathbf{S}$ & $\mathbf{S}$ & $\mathbf{U}$ & $\mathbf{U}$ & $\mathbf{S}$ & $\mathbf{S}$ & $\mathbf{S}$ \\
\hline \multirow{2}{*}{ HLLE } & $1^{\text {st }}$ & $\mathbf{S}$ & $\mathbf{S}$ & $\mathbf{S}$ & $\mathbf{U}$ & $\mathbf{U}$ & $\mathbf{S}$ & $\mathbf{S}$ & $\mathbf{S}$ & $\mathbf{S}$ \\
\hline & $2^{\text {nd }}$ & $\mathbf{S}$ & $\mathbf{S}$ & $\mathbf{S}$ & $\mathbf{U}$ & $\mathbf{U}$ & $\mathbf{U}$ & $\mathbf{S}$ & $\mathbf{S}$ & $\mathbf{S}$ \\
\hline
\end{tabular}

According to Table 2, the following discussions have been drawn:

- All the schemes presented here showed 'Unstable (U)' or 'Asymmetric (A)' results for some conditions. In every case we find some set of consecutive positions for which the solution is 'Stable (S),' and another set for which it is 'Unstable (U).' Sometimes these sets are separated by an example of case 'A.' This behavior was also noted in the $1 \mathrm{D}$ and $11 / 2 \mathrm{D}$ tests.

- The boundaries between 'Stable' and the other results are different for different schemes.

- The boundaries are also different for different order of spatial accuracies (e.g., the new scheme with $\alpha=0.2$ favors $3 / 8 \leq \delta \leq 6 / 8$ for $1^{\text {st }}$-order and $4 / 8 \leq \delta \leq 7 / 8$ for $2^{\text {nd }}$-order). This difference would be due to difference of computed shock stand-off distances.

- The new scheme with $\alpha=0.8$ failed all the cases, in contrast to $1 \mathrm{D}$ tests but as in $11 / 2 \mathrm{D}$ cases. It seemed very vulnerable to multidimensional effects.

- "Entropy-fix" slightly helped Roe scheme to be stable for $2^{\text {nd }}$-order, but not for $1^{\text {st }}$-order.

- AUSM+ has the smallest 'Unstable' region. This seems to be because the scheme has gentle growth rate of instability as shown in $11 / 2 \mathrm{D}$ tests, and the solution converged before such instability reached significant amount in most of 'Stable' cases.

- Schemes equipped with multidimensional effects (AUSMPW+ and RoeM2) still suffered from shock instablility.

11 of 21

American Institute of Aeronautics and Astronautics 
These results are broadly similar to the $11 / 2 \mathrm{D}$ results. For most of the schemes, the proportions of stable and unstable cases were about the same. Unfortunately, there is again no stable scheme.

Readers who are interested in detailed computed flowfields and residual histories may refer to Figs. 11-28.

\section{Conclusions}

We have conducted a broad range of investigations of hypersonic shock stability within the common framework of upwind shock-capturing schemes. We have focused on the role played by the relative positioning of the shock on the grid. All but two of the flux functions investigated were unstable, even in one dimension, for at least some combinations of shock location and freestream Mach number. Although we believe that passing this test is a necessary condition for a flux function to be reliable in more general settings it is far from sufficient. We think it likely that some form of multidimensional dissipation is required, which may take the form of a dissipation added to a finite-volume method, or a more radically multidimensional formulation. On the limited basis of the present tests, the dissipation due to K.H. Kim et al. in the context of AUSMPW+ seems more reliable than that due to S.S. Kim et $a l$. for RoeM2. However, neither is formulated for use on unstructured grids.

Our immediate plans involve determining a more rational basis for choosing the parameter $\alpha$ in the new scheme, and designing a form of dissipation that will apply to unstructured grids.

\section{Acknowledgments}

We thank Dr. Jeffery White, NASA Langley for supplying us a computational grid for a Mach 6 blunt-body problem in Section IV-C. We also thank Professor Nakamura and his colleagues at Nagoya University, and Professor Powell, University of Michigan for their support for computational resources.

The first author is grateful to the Student Exchange program between University of Michigan and Nagoya University, and to Professor Van Leer, University of Michigan for the relevant support.

\section{References}

${ }^{1}$ Godunov S.K., "A finite difference method for the numerical computation of discontinuous solutions of the equations of fluid dynamics," Mat. Sb., 47, p.271, 1959.

2 P.L. Roe, “Approximate Riemann Solvers, Parameter Vectors, and Difference Schemes,” J. Comp. Phys., 43, pp.357-372, 1981.

${ }^{3}$ Kim, S.S., Kim, C., Rho, O.H., Hong, S.K., "Cures for the shock instability, Development of a shock-stable Roe scheme," $J$. Comp. Phys., 186, p.342, 2003.

${ }^{4}$ M.S. Liou, "A sequel to AUSM: AUSM+," J. Comp. Phys., 129, p.364, 1996.

${ }^{5}$ M.S. Liou, “A sequel to AUSM, Part II: AUSM+-up for all speeds,” J. Comp. Phys., 214, p.137, 2006.

${ }^{6}$ B. Einfeldt, "On Godunov-Type Methods for Gas Dynamics," SIAM J. Numer. Anal., 25(2), 1988.

${ }^{7}$ Kim, K.H., Kim, C., and Rho, O.H., "Methods for the Accurate Computations of Hypersonic Flows I. AUSMPW+ scheme," J. Comp. Phys., 174, pp.38-80, 2001.

${ }^{8}$ F. Ismail, Ph.D. Dissertation, University of Michigan, 2006.

${ }^{9}$ F. Ismail, P.L. Roe, and H. Nishikawa, "A Proposed Cure to the Carbuncle Phenomenon," $4^{\text {th }}$ ICCFD, 2006.

${ }^{10}$ Barth, T.J., "Some Notes on Shock-Resolving Flux Functions Part I: Stationary Characteristics,” NASA TM-101087, 1989.

${ }^{11}$ A. Harten, "High Resolution Schemes for Hyperbolic Conservation Laws," J. Comp. Phys., 49, p.357, 1983.

12 Roe P.L., "Fluctuations and signals-a framework for numerical evolution problems," Numerical Methods for Fluid Dynamics, Edited by Morton KW and Baines MJ, pp.232-236, 1982.

${ }^{13}$ Chauvat, Y., Moschetta, J.M., and Gressier J., "Shock wave numerical structure and the carbuncle phenomenon," Int. J. Num. Meth. Fluids, 47, pp.903-909, 2005.

${ }_{14}^{14}$ J.J. Quirk, “A Contribution to the Great Rimann Solver Debate,” Int. J. Num. Meth. Fluids, 18, p.555, 1994.

${ }^{15}$ M. Dumbser, J.M. Moschetta, J. Gressier, “A matrix stability analysis of the carbuncle phenomenon,” J. Comp. Phys., 197, p.647, 2004.

${ }^{16}$ P.L. Roe, H. Nishikawa, F. Ismail and L. Scalabrin, “On Carbuncles and Other Excrescences,” AIAA Paper 2005-4872, 2005.

17 Sanders, R., Morano, E. and Druguetz, M.C., "Multidimensional Dissipation for Upwind Schemes: Stability and Applications to Gas Dynamics," J. Comp. Phys., 145, pp.511-537, 1998

${ }^{18}$ Ren, Y.X., "A robust shock-capturing scheme based on rotated Riemann solvers," Computers \& Fluids., 32, pp. 13791403, 2003.

${ }^{19}$ Van Leer, B., "Towards the Ultimate Conservative Difference Scheme. V. A Second-Order Sequel to Godunov's Method," J. Comp. Phys., 32, pp.101-136, 1979.

${ }^{20}$ Van Albada, G.D., Van Leer, B. and Roberts, Jr., W.W., “A Comparative Study of Computational Methods in Cosmic Gas Dynamics," Astron. Astrophys., 108, pp.76-84, 1982. 


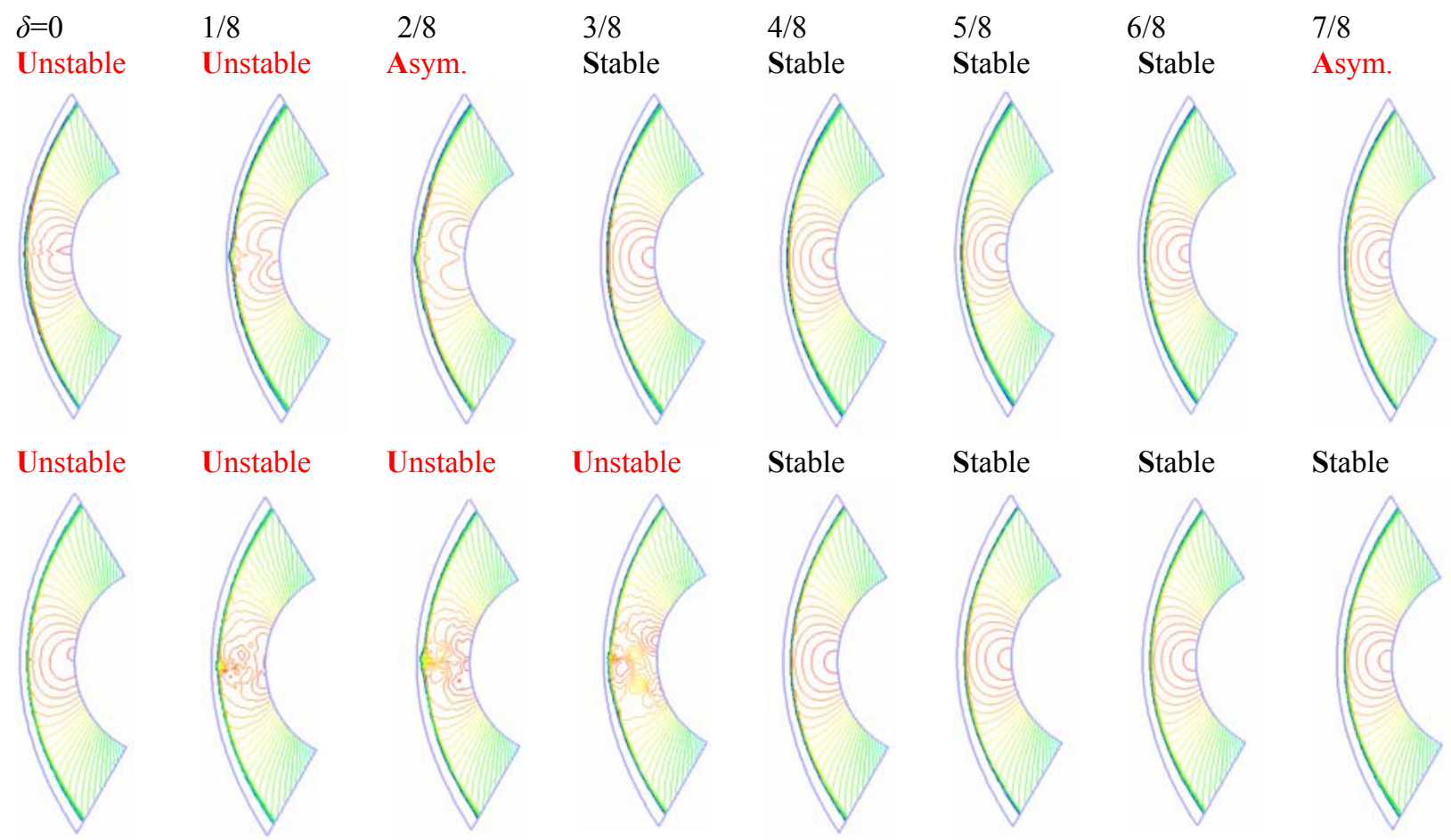

Figure 11. Pressure Coefficient Contours (Upper: $1^{\text {st }}$-order; Lower: $2^{\text {nd }}$-order) around 2D Cylinder at $50,000$ time steps (New, $\alpha=0.2)$.

(a) $1^{\text {st }}$-order, $0 \leq \delta \leq 3 / 8$

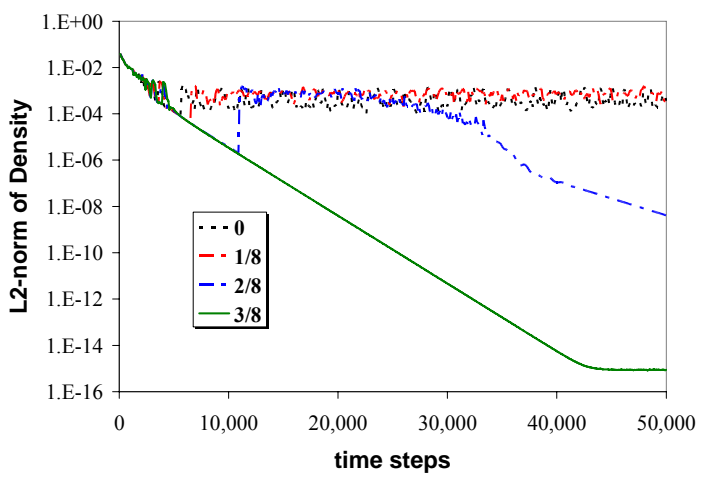

(c) $2^{\text {nd }}$-order, $0 \leq \delta \leq 3 / 8$

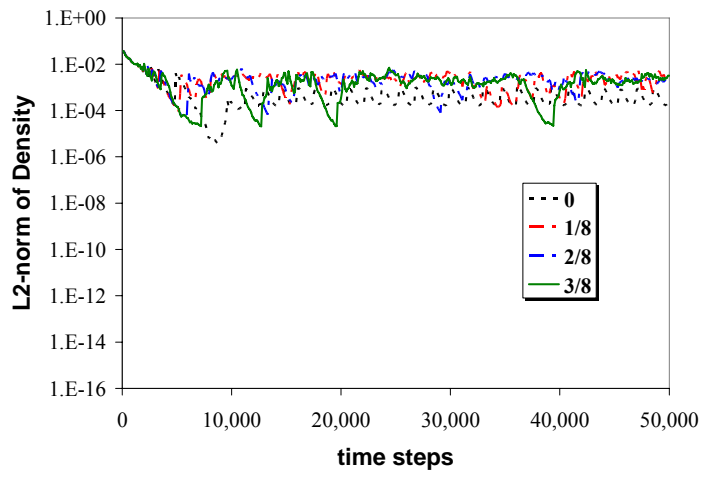

(b) $1^{\text {st }}$-order, $4 / 8 \leq \delta \leq 7 / 8$

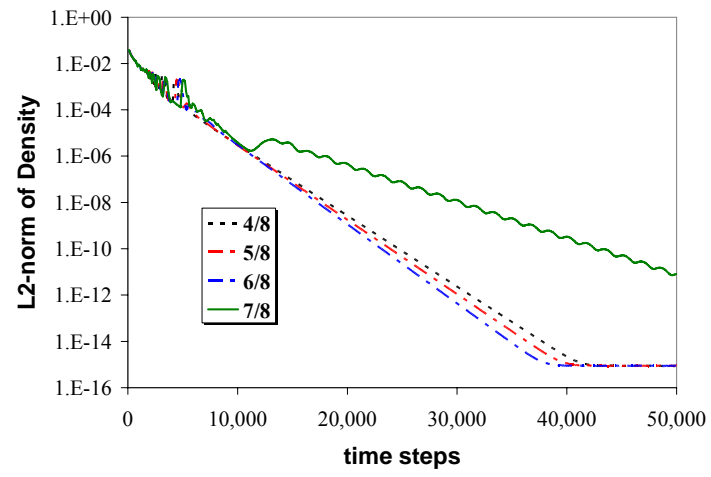

(d) $2^{\text {nd }}$-order, $4 / 8 \leq \delta \leq 7 / 8$

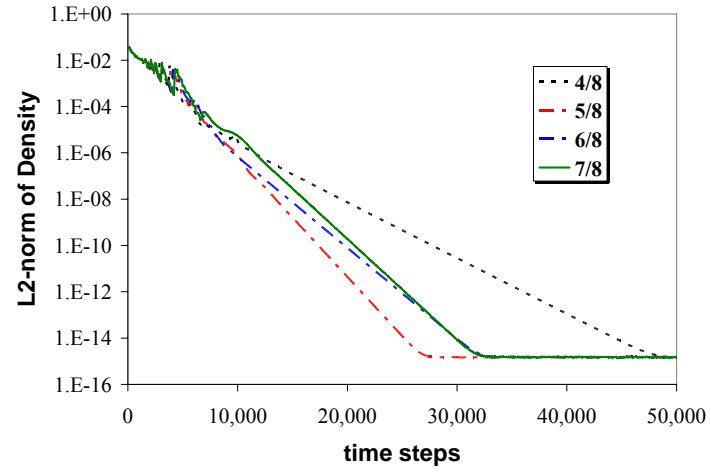

Figure 12. Residual Histories for Two Dimensional Cylinder Problem (New, $\alpha=0.2$ ). 


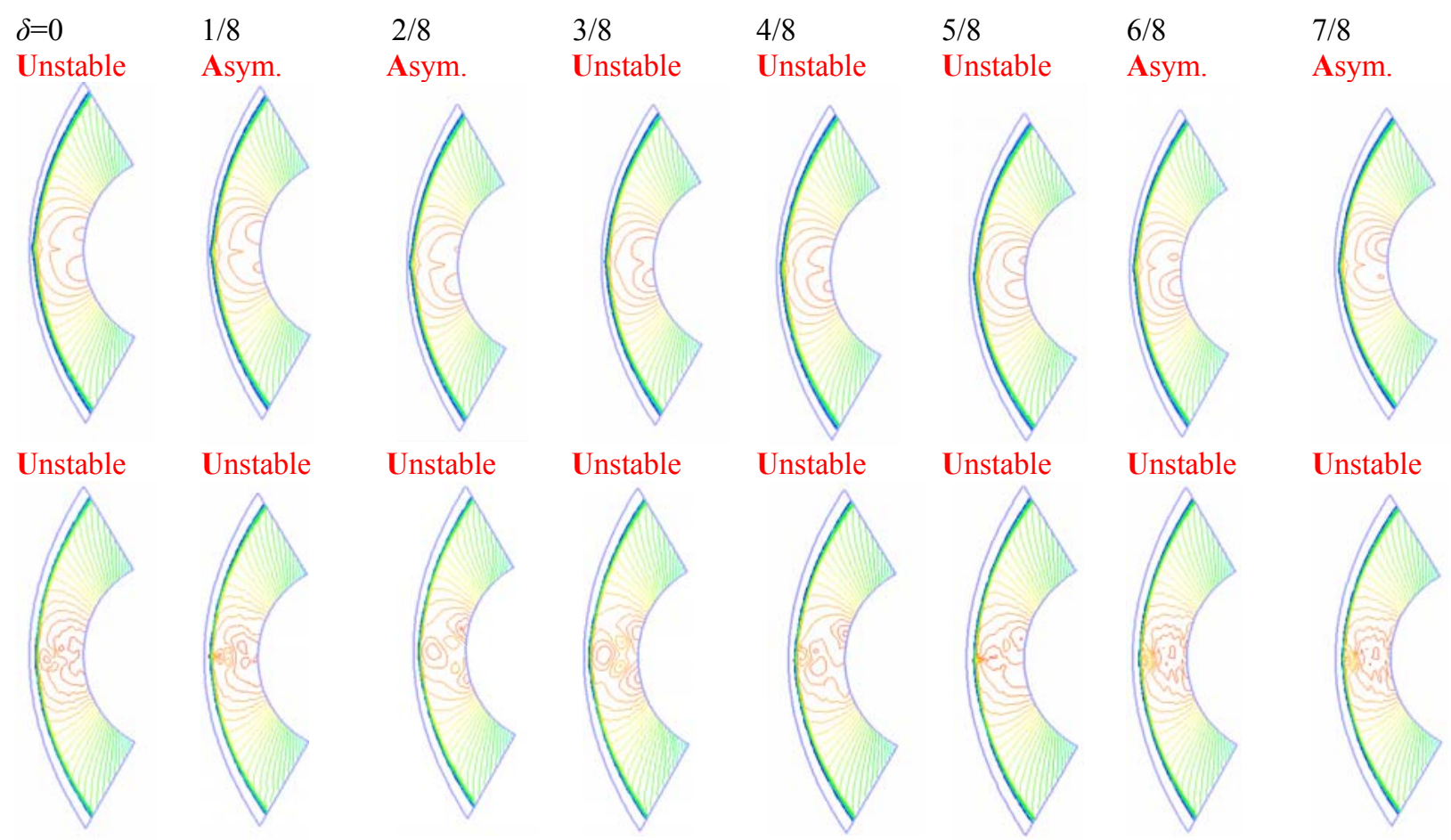

Figure 13. Pressure Coefficient Contours (Upper: $\mathbf{1}^{\text {st }}$-order; Lower: $\mathbf{2}^{\text {nd }}$-order) around 2D Cylinder at 50,000 time steps (New, $\alpha=0.8$ ).

(a) $1^{\text {st }}$-order, $0 \leq \delta \leq 3 / 8$

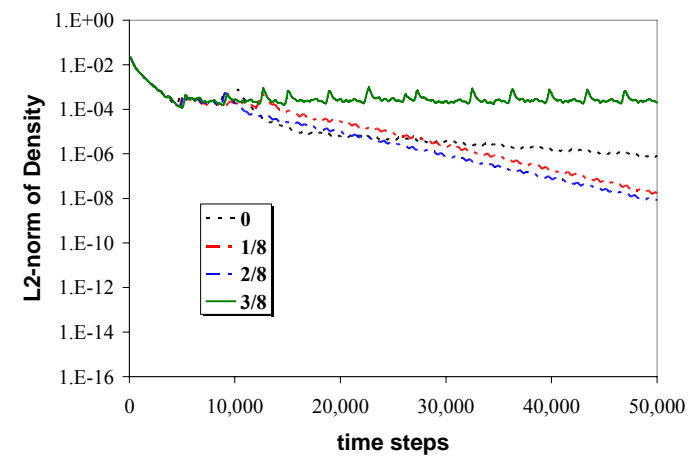

(c) $2^{\text {nd }}$-order, $0 \leq \delta \leq 3 / 8$

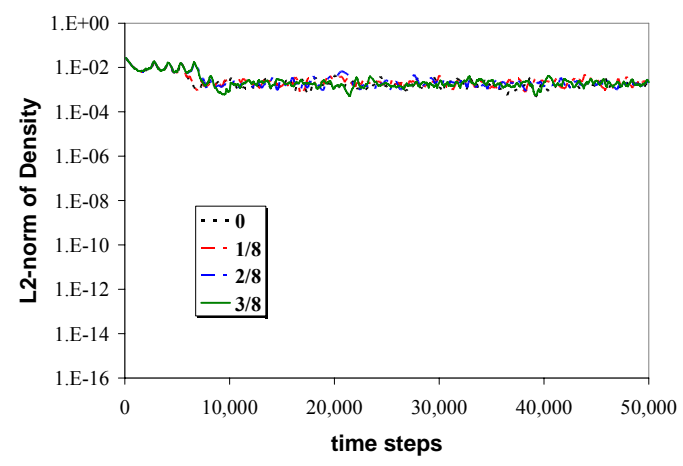

(b) $1^{\text {st }}$-order, $4 / 8 \leq \delta \leq 7 / 8$

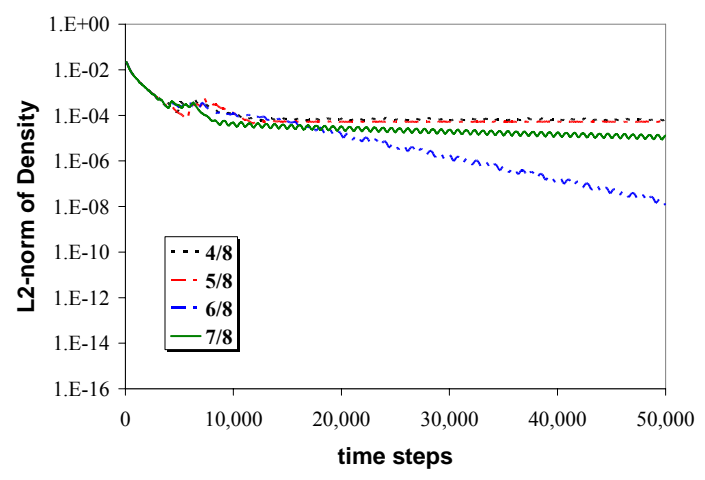

(d) $2^{\text {nd }}$-order, $4 / 8 \leq \delta \leq 7 / 8$

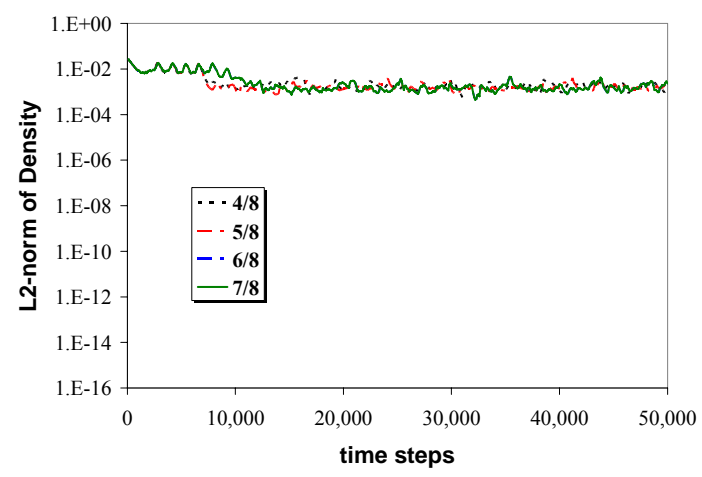

Figure 14. Residual Histories for Two Dimensional Cylinder Problem (New, $\alpha=0.8$ ). 


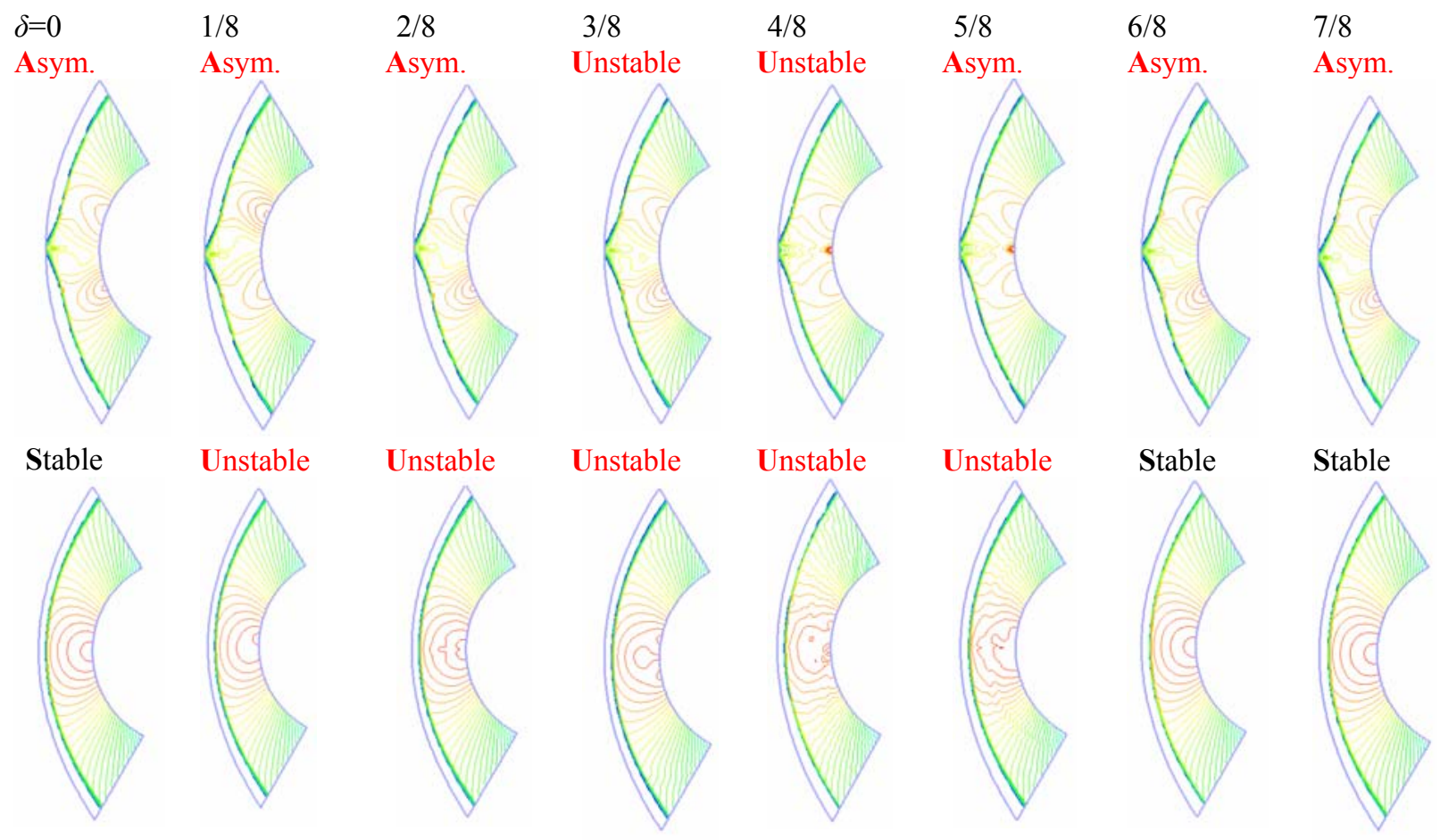

Figure 15. Pressure Coefficient Contours (Upper: $1^{\text {st }}$-order; Lower: $\mathbf{2}^{\text {nd }}$-order) around $2 \mathrm{D}$ Cylinder at 50,000 time steps (Roe).

(a) $1^{\text {st }}$-order, $0 \leq \delta \leq 3 / 8$

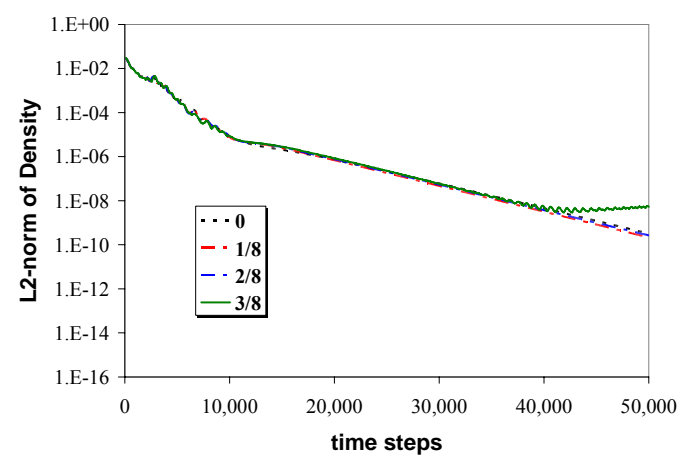

(c) $2^{\text {nd }}$-order, $0 \leq \delta \leq 3 / 8$

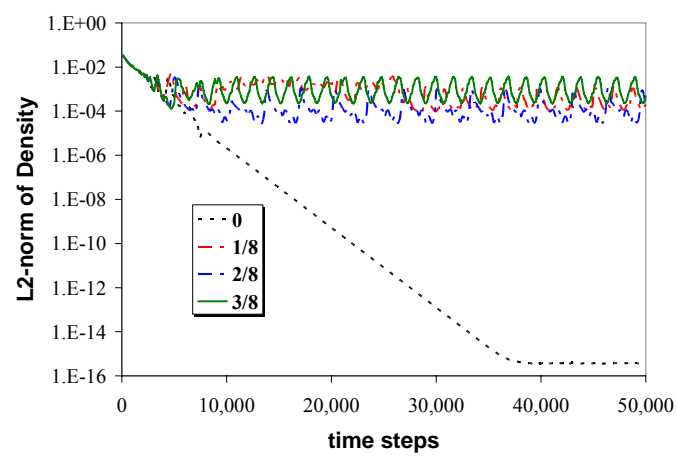

(b) $1^{\text {st }}$-order, $4 / 8 \leq \delta \leq 7 / 8$

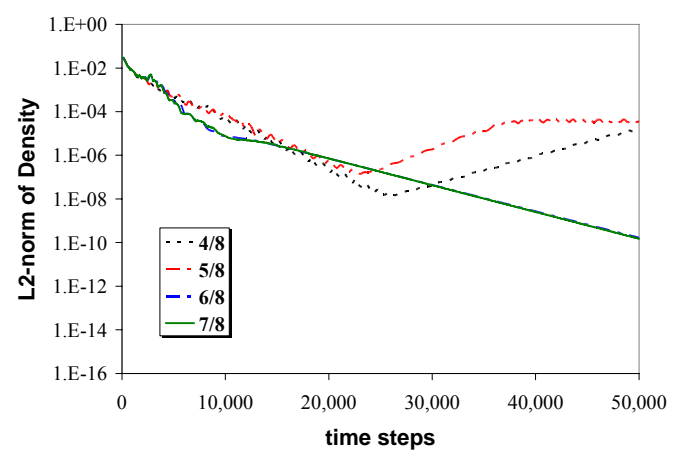

(d) $2^{\text {nd }}$-order, $4 / 8 \leq \delta \leq 7 / 8$

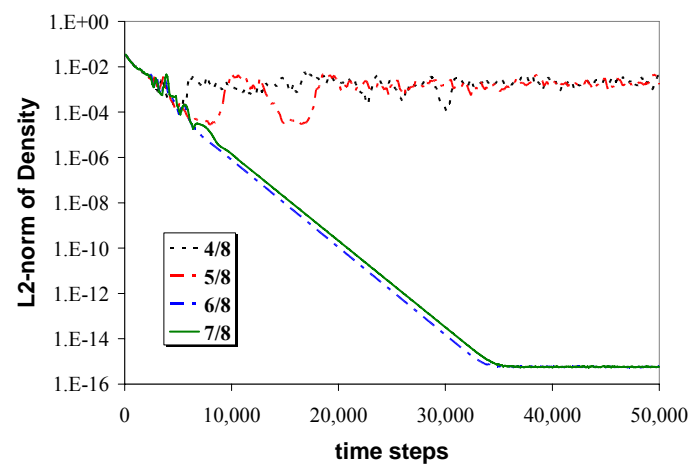

Figure 16. Residual Histories for Two Dimensional Cylinder Problem (Roe). 


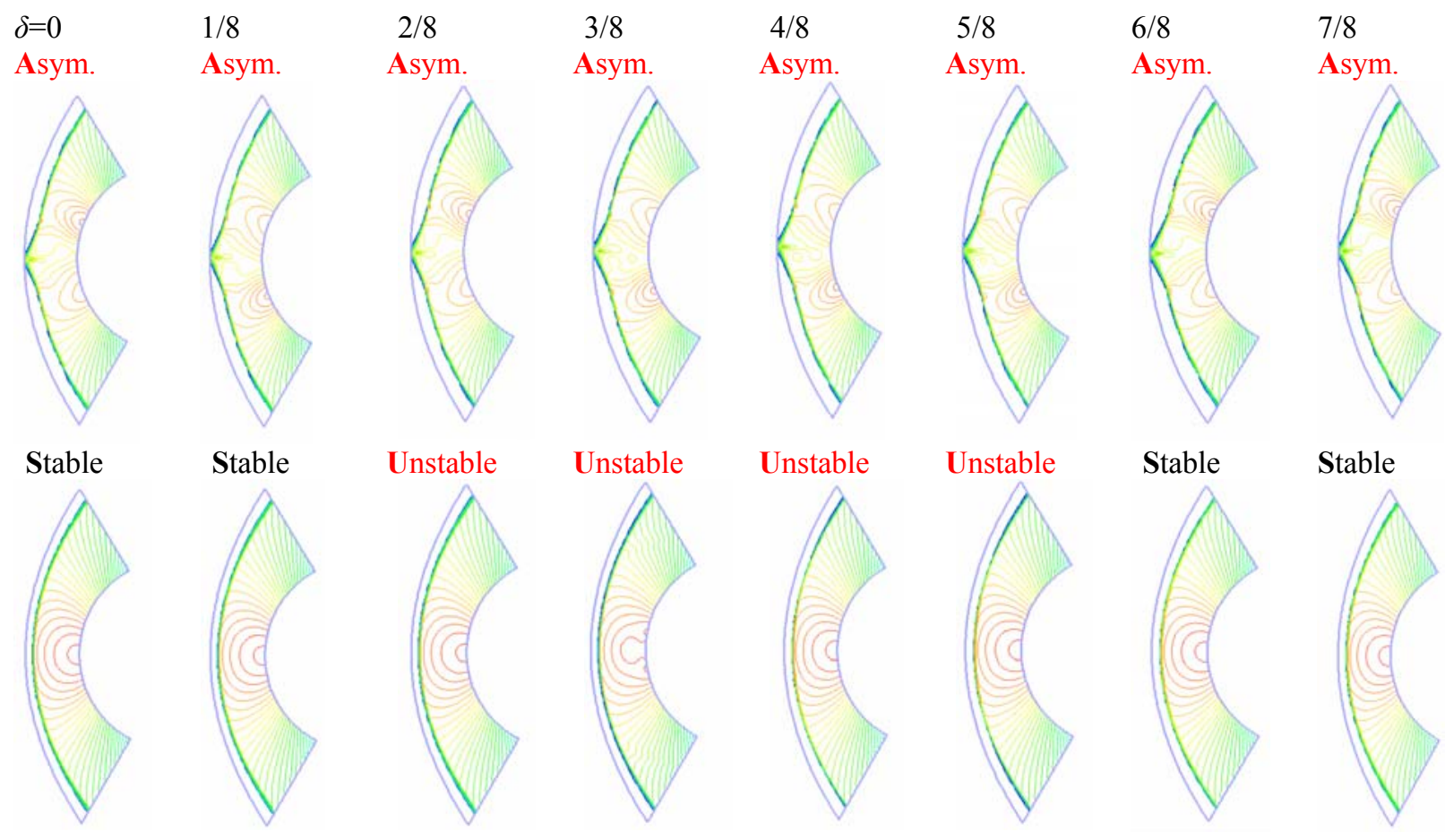

Figure 17. Pressure Coefficient Contours (Upper: $\mathbf{1}^{\text {st }}$-order; Lower: $2^{\text {nd }}$-order) around 2D Cylinder at $\mathbf{5 0 , 0 0 0}$ time steps (Roe, E-Fix).

(a) $1^{\text {st }}$-order, $0 \leq \delta \leq 3 / 8$

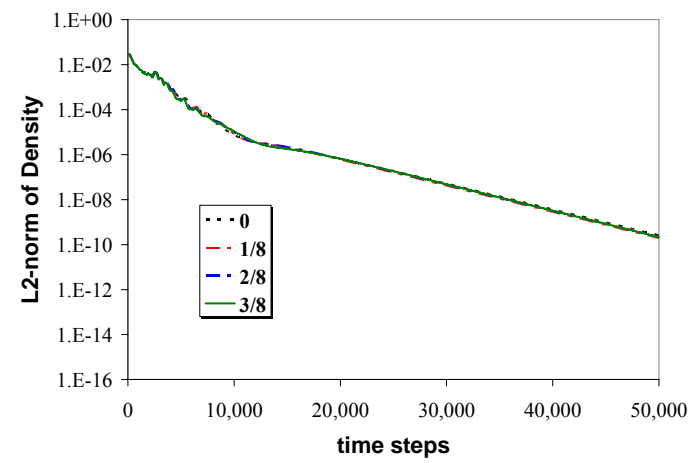

(c) $2^{\text {nd }}$-order, $0 \leq \delta \leq 3 / 8$

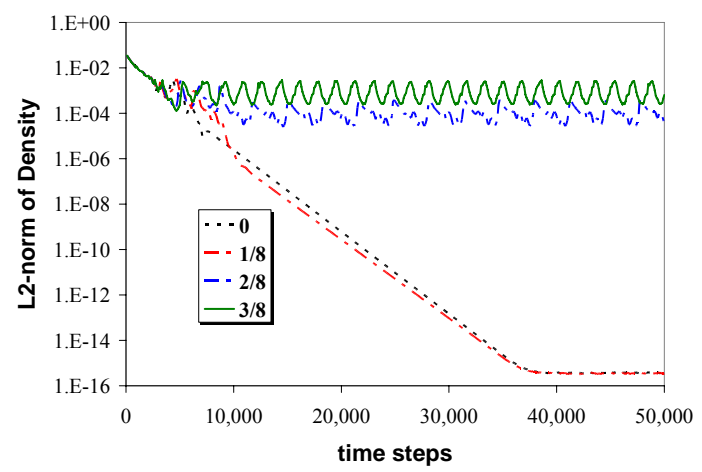

(b) $1^{\text {st }}$-order, $4 / 8 \leq \delta \leq 7 / 8$

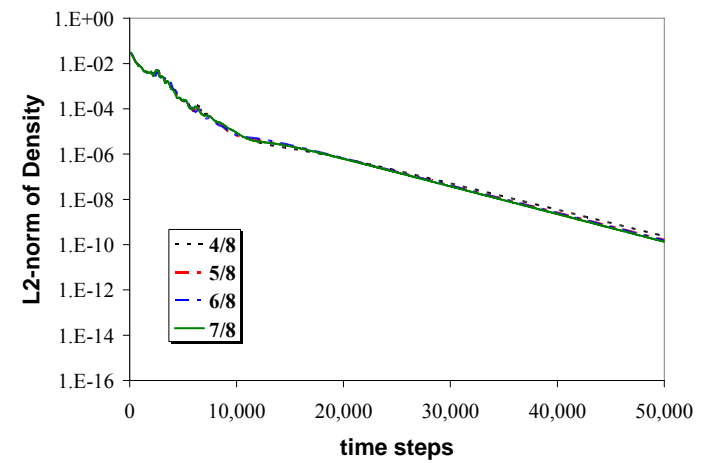

(d) $2^{\text {nd }}$-order, $4 / 8 \leq \delta \leq 7 / 8$

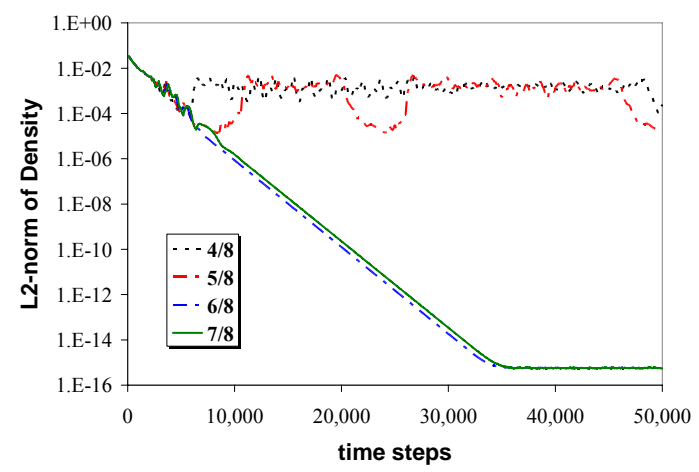

Figure 18. Residual Histories for Two Dimensional Cylinder Problem (Roe, E-Fix). 


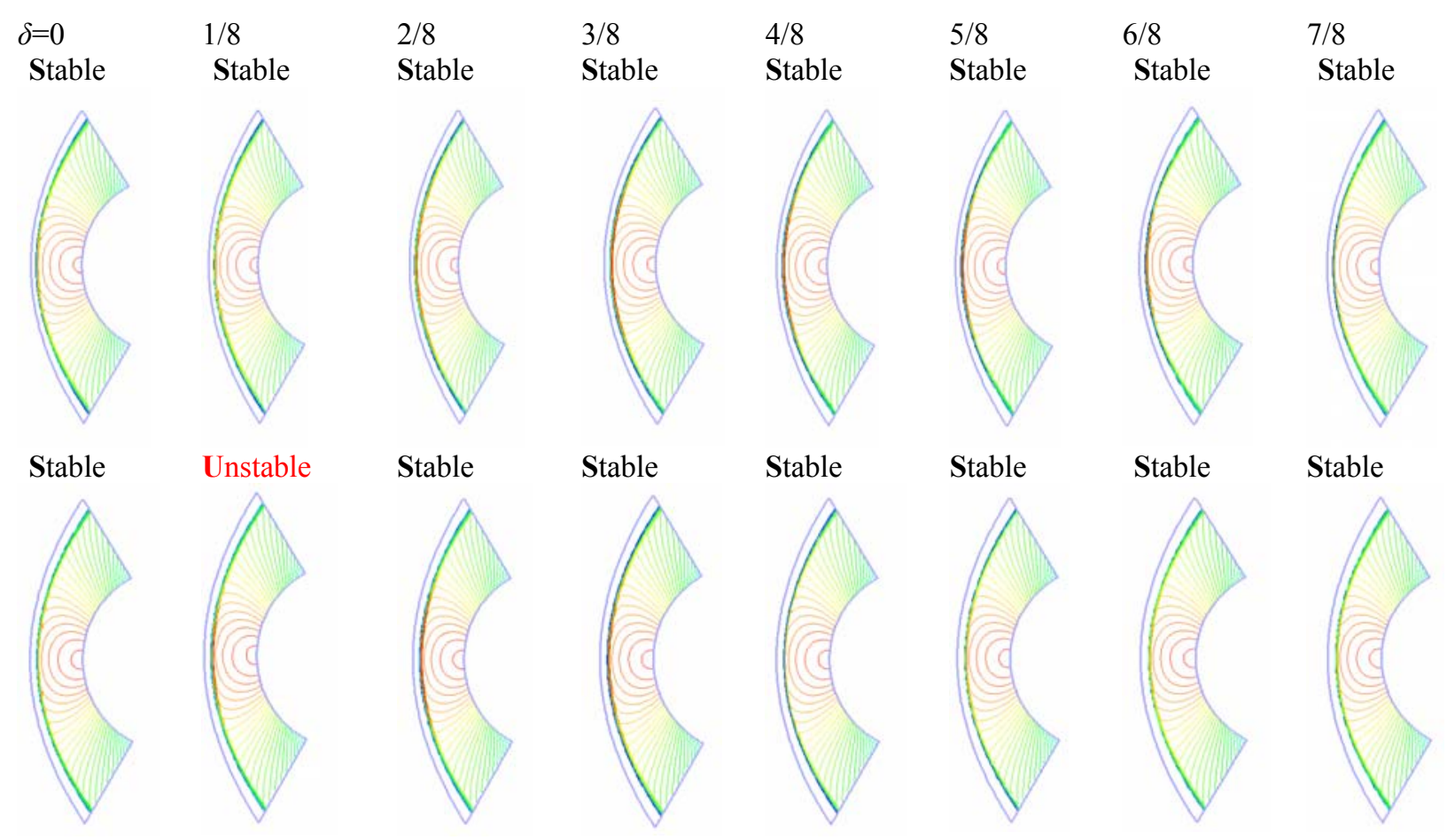

Figure 19. Pressure Coefficient Contours (Upper: $1^{\text {st }}$-order; Lower: $2^{\text {nd }}$-order) around 2D Cylinder at $\mathbf{5 0 , 0 0 0}$ time steps (AUSM+).

(a) $1^{\text {st }}$-order, $0 \leq \delta \leq 3 / 8$

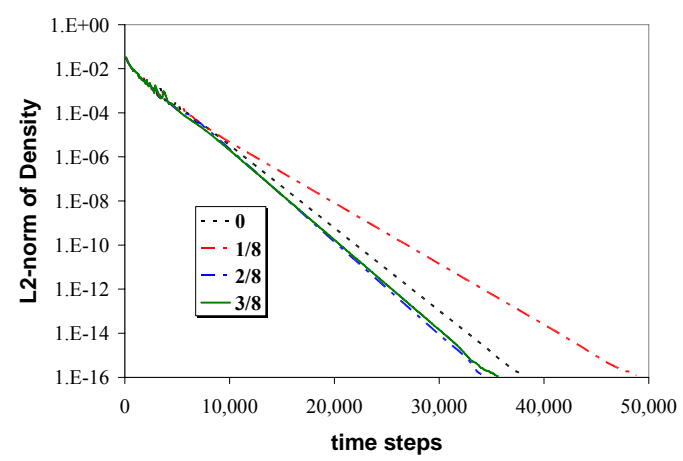

(c) $2^{\text {nd }}$-order, $0 \leq \delta \leq 3 / 8$

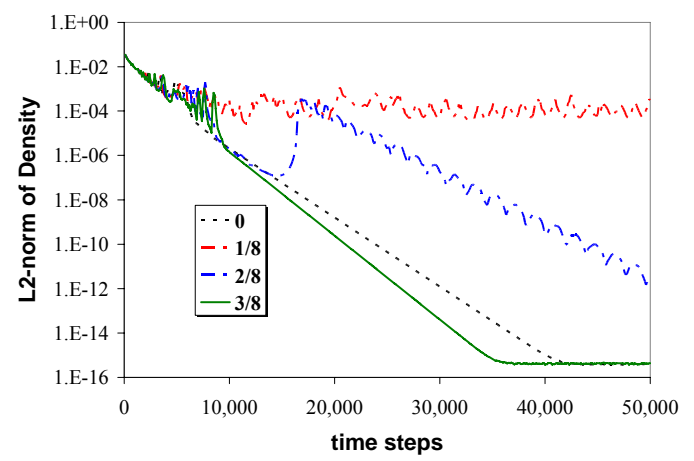

(b) $1^{\text {st }}$-order, $4 / 8 \leq \delta \leq 7 / 8$

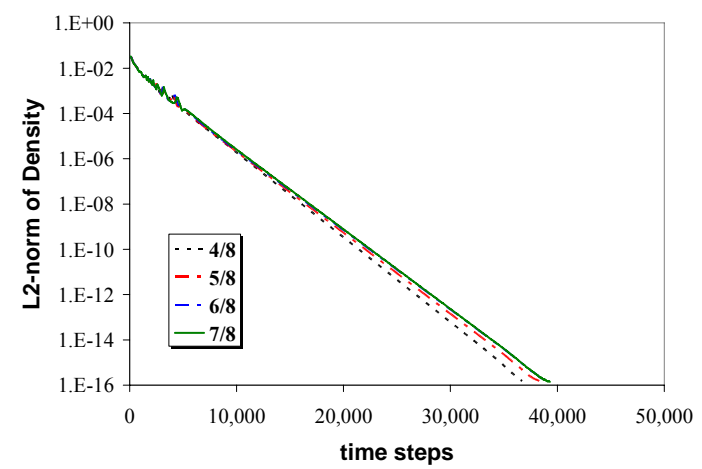

(d) $2^{\text {nd }}$-order, $4 / 8 \leq \delta \leq 7 / 8$

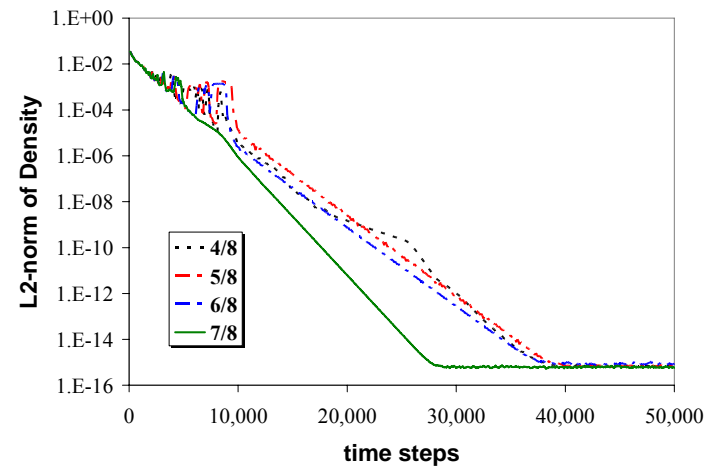

Figure 20. Residual Histories for Two Dimensional Cylinder Problem (AUSM+). 


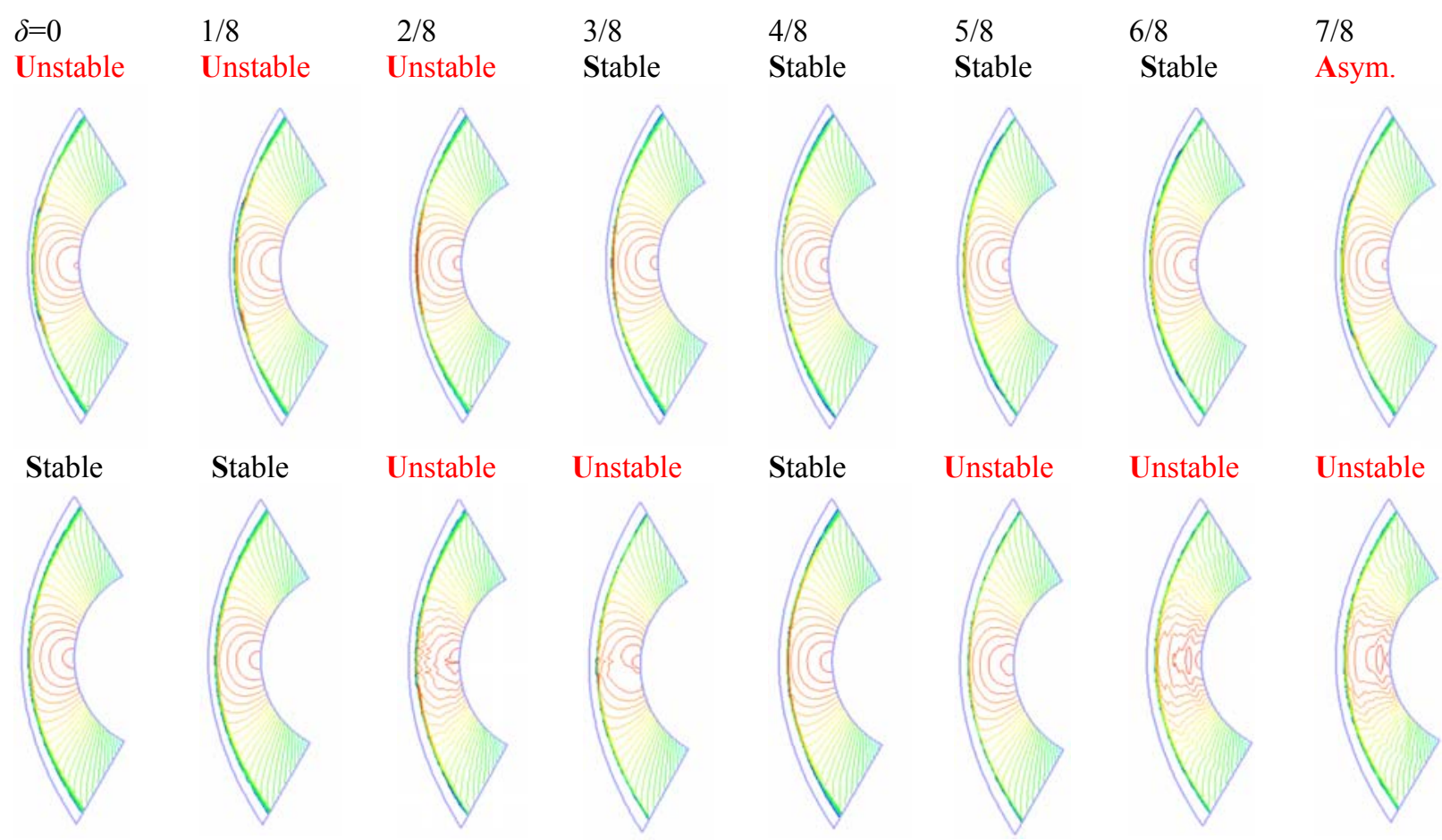

Figure 21. Pressure Coefficient Contours (Upper: $\mathbf{1}^{\text {st }}$-order; Lower: $\mathbf{2}^{\text {nd }}$-order) around 2D Cylinder at 50,000 time steps (AUSM+ ${ }^{+}$up).

(a) $1^{\text {st }}$-order, $0 \leq \delta \leq 3 / 8$

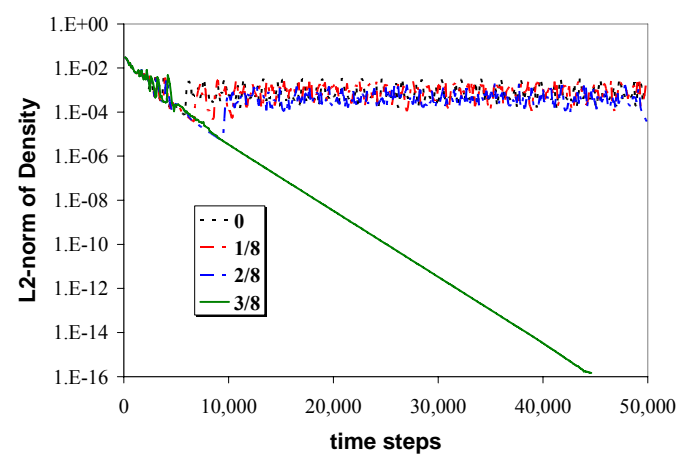

(c) $2^{\text {nd }}$-order, $0 \leq \delta \leq 3 / 8$

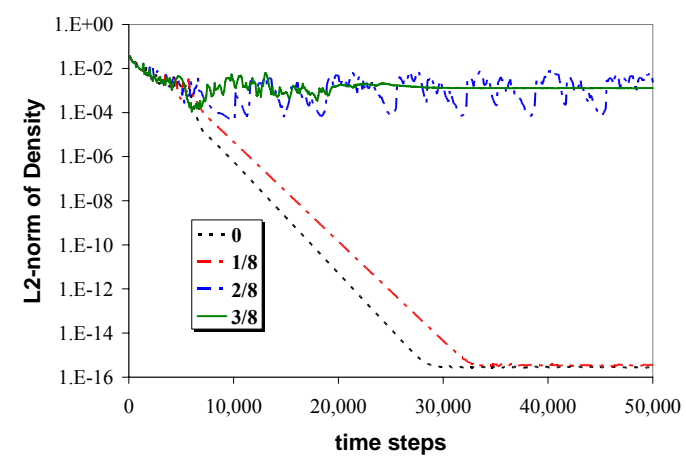

(b) $1^{\text {st }}$-order, $4 / 8 \leq \delta \leq 7 / 8$

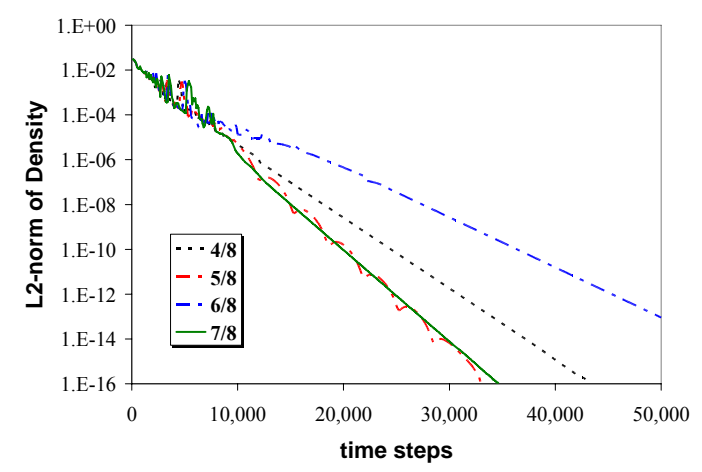

(d) $2^{\text {nd }}$-order, $4 / 8 \leq \delta \leq 7 / 8$

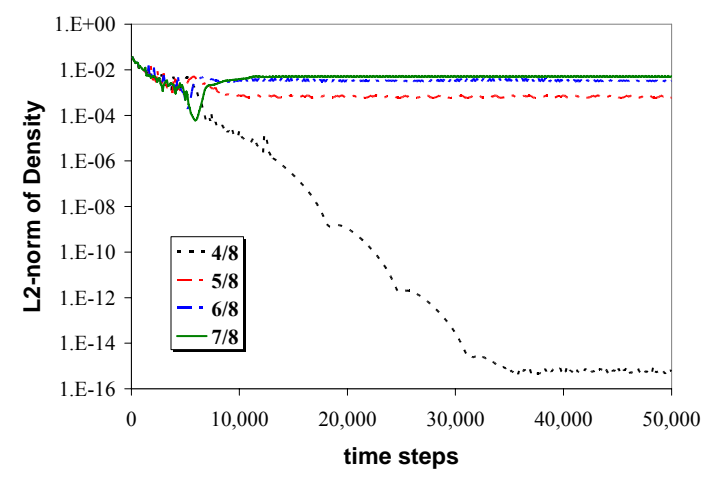

Figure 22. Residual Histories for Two Dimensional Cylinder Problem (AUSM+-up). 


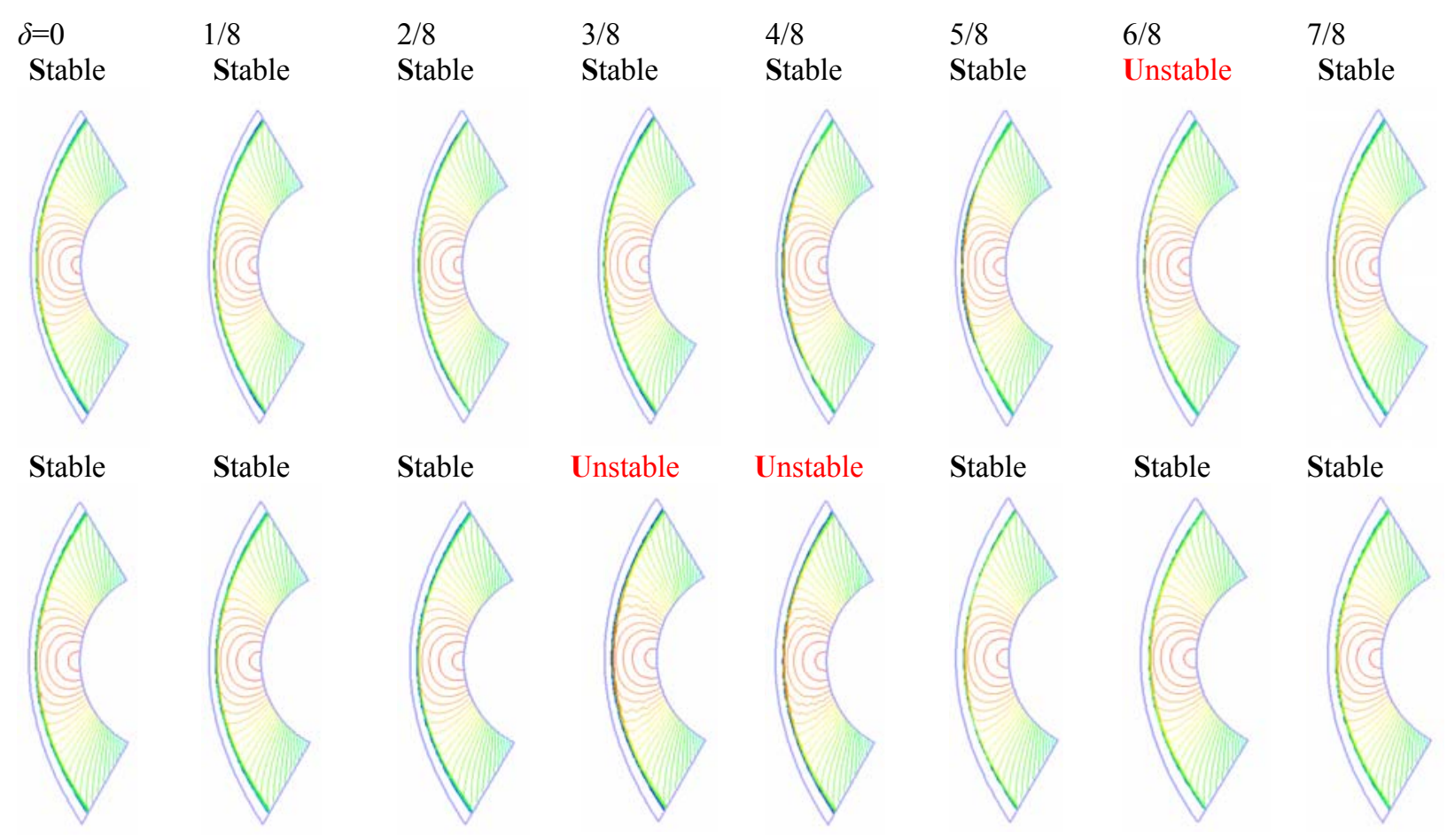

Figure 23. Pressure Coefficient Contours (Upper: $1^{\text {st }}$-order; Lower: $2^{\text {nd }}$-order) around 2D Cylinder at 50,000 time steps (AUSMPW+).

(a) $1^{\text {st }}$-order, $0 \leq \delta \leq 3 / 8$

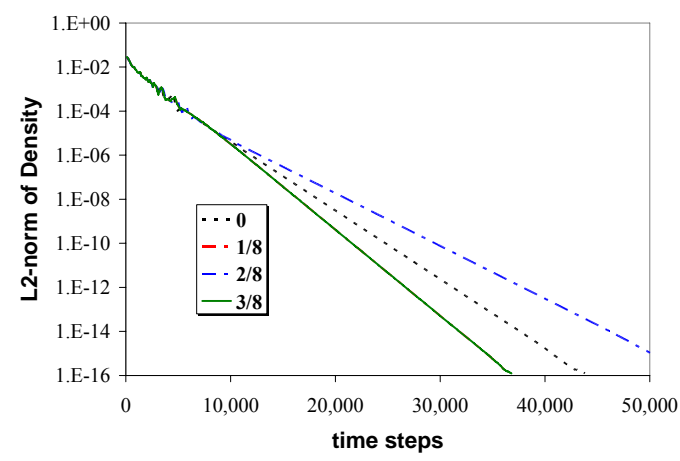

(c) $2^{\text {nd }}$-order, $0 \leq \delta \leq 3 / 8$

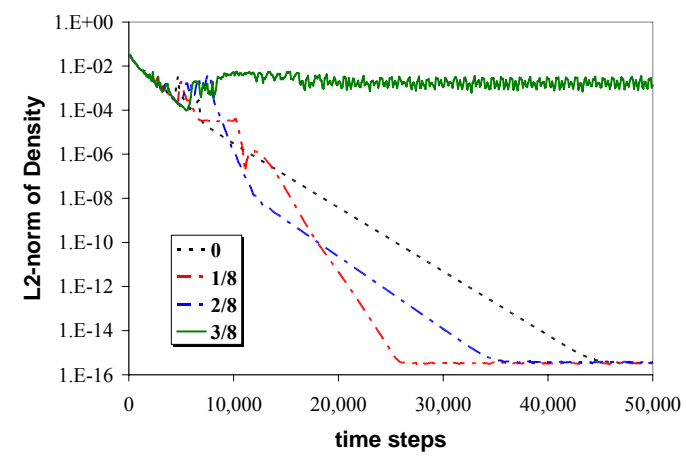

(b) $1^{\text {st }}$-order, $4 / 8 \leq \delta \leq 7 / 8$

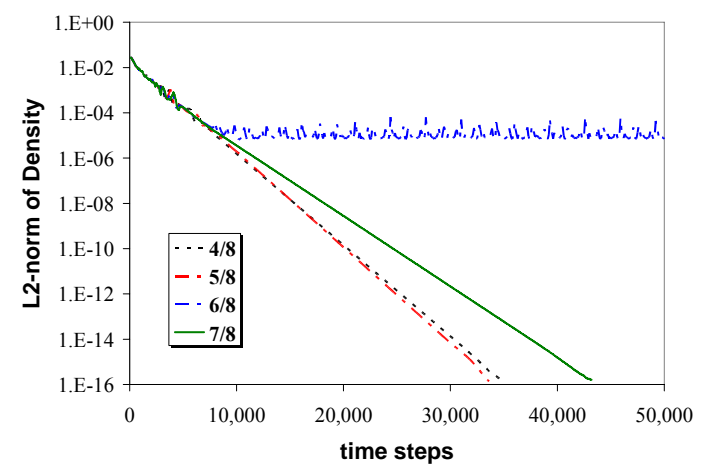

(d) $2^{\text {nd }}$-order, $4 / 8 \leq \delta \leq 7 / 8$

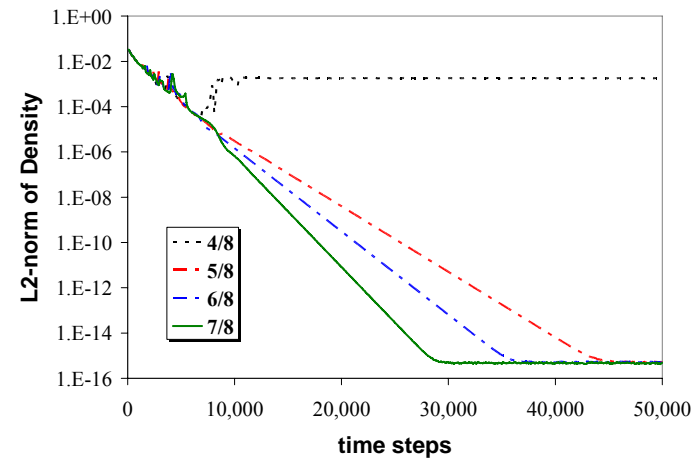

Figure 24. Residual Histories for Two Dimensional Cylinder Problem (AUSMPW+). 


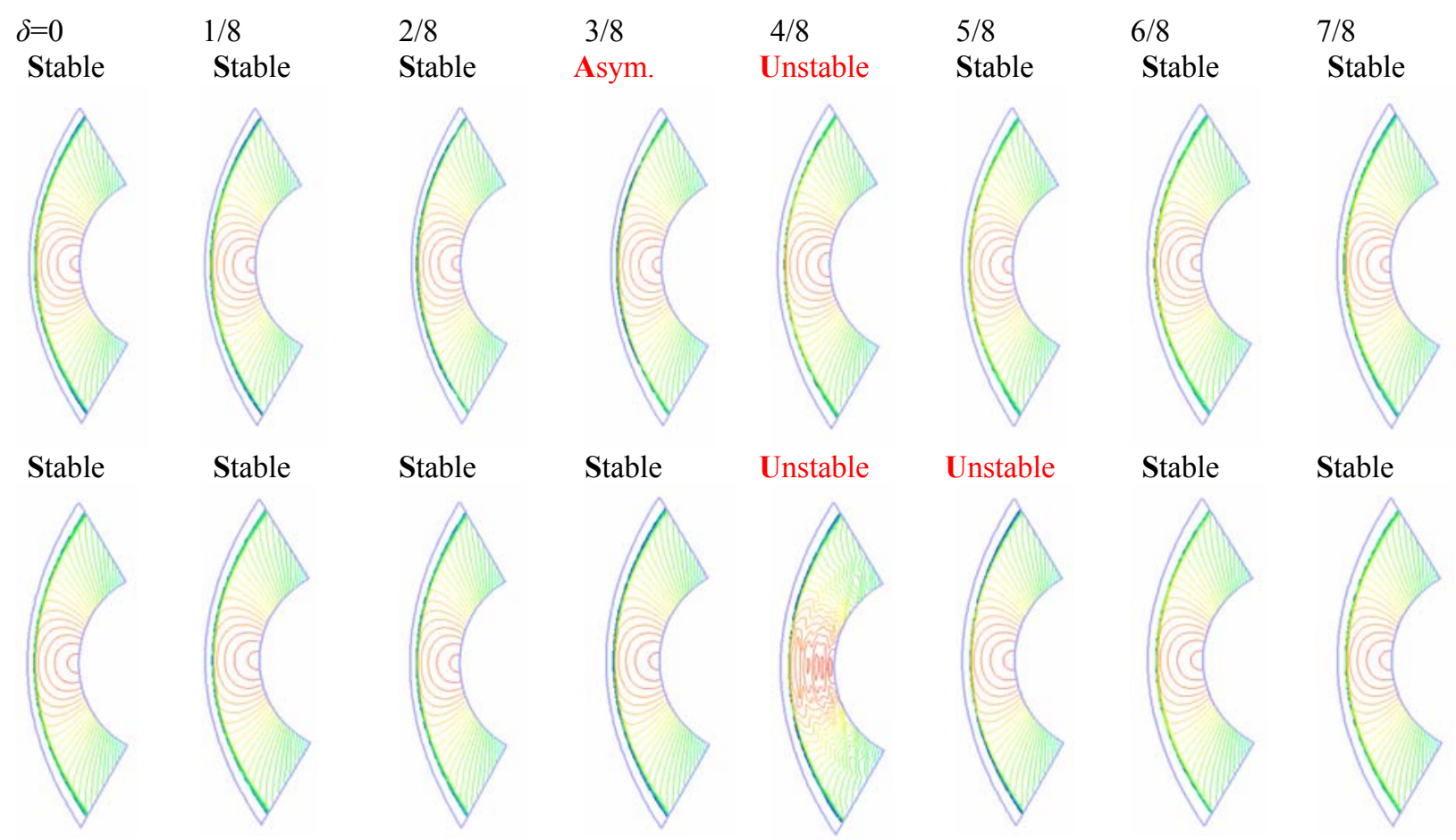

Figure 25. Pressure Coefficient Contours (Upper: ${ }^{\text {st }}$-order; Lower: $2^{\text {nd }}$-order) around 2D Cylinder at 50,000 time steps (RoeM2).

(a) $1^{\text {st }}$-order, $0 \leq \delta \leq 3 / 8$

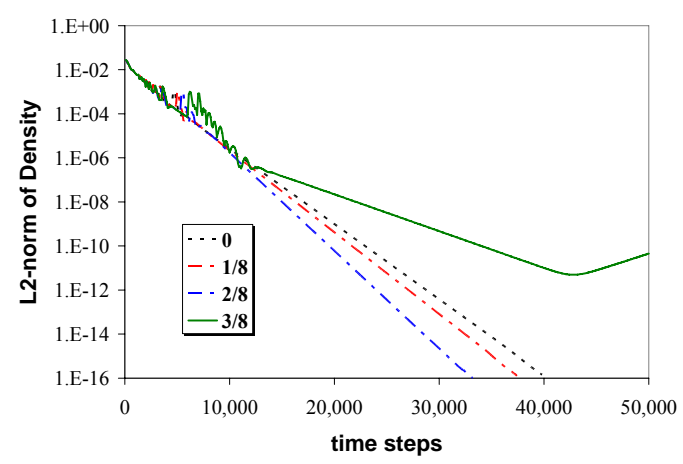

(c) $2^{\text {nd }}$-order, $0 \leq \delta \leq 3 / 8$

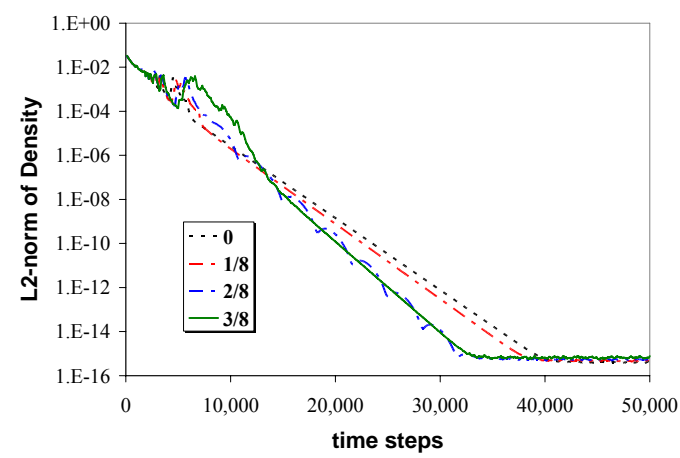

(b) $1^{\text {st }}$-order, $4 / 8 \leq \delta \leq 7 / 8$

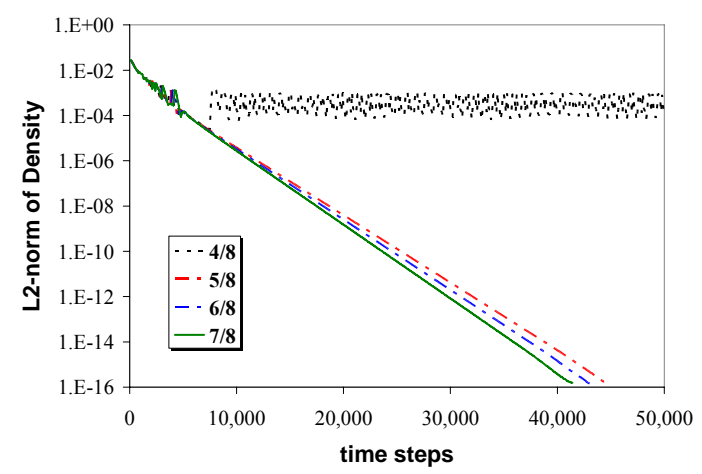

(d) $2^{\text {nd }}$-order, $4 / 8 \leq \delta \leq 7 / 8$

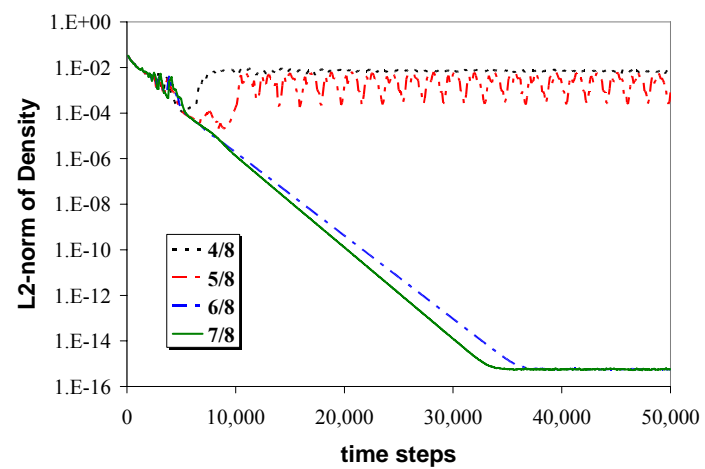

Figure 26. Residual Histories for Two Dimensional Cylinder Problem (RoeM2). 


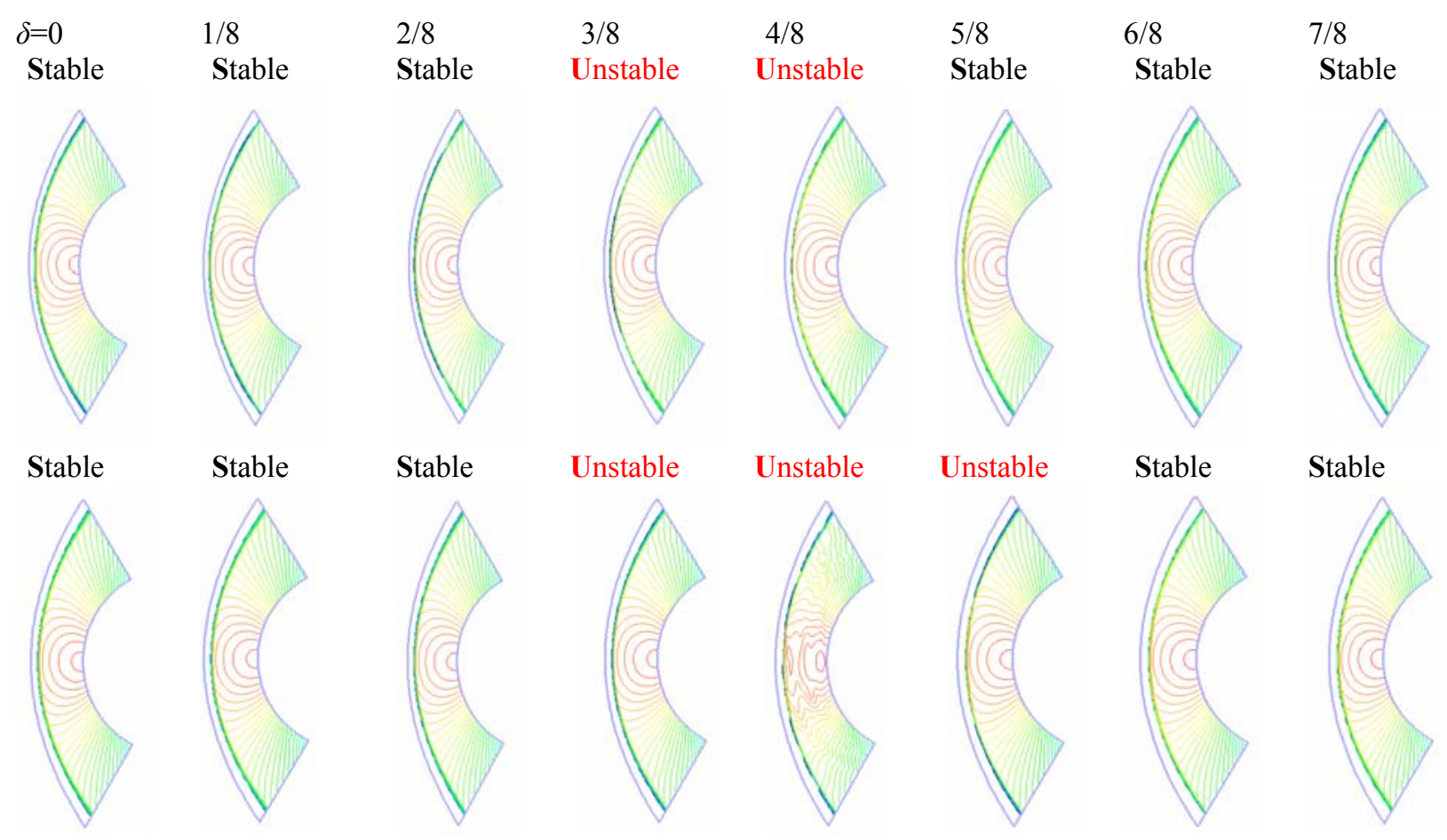

Figure 27. Pressure Coefficient Contours (Upper: $1^{\text {st }}$-order; Lower: $2^{\text {nd }}$-order) around 2D Cylinder at 50,000 time steps (HLLE).

(a) $1^{\text {st }}$-order, $0 \leq \delta \leq 3 / 8$

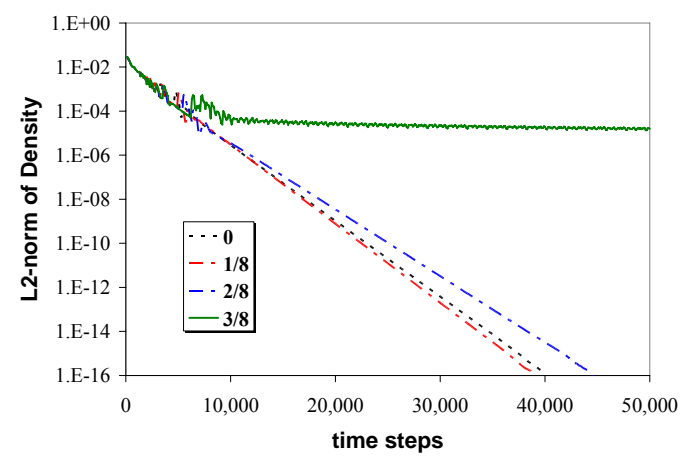

(c) $2^{\text {nd }}$-order, $0 \leq \delta \leq 3 / 8$

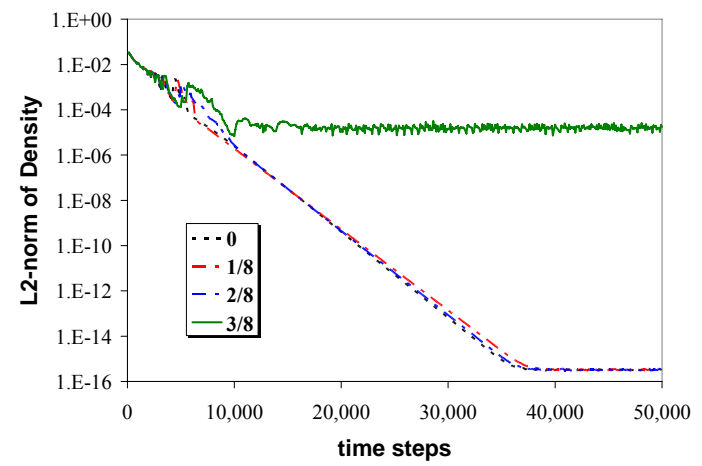

(b) $1^{\text {st }}$-order, $4 / 8 \leq \delta \leq 7 / 8$

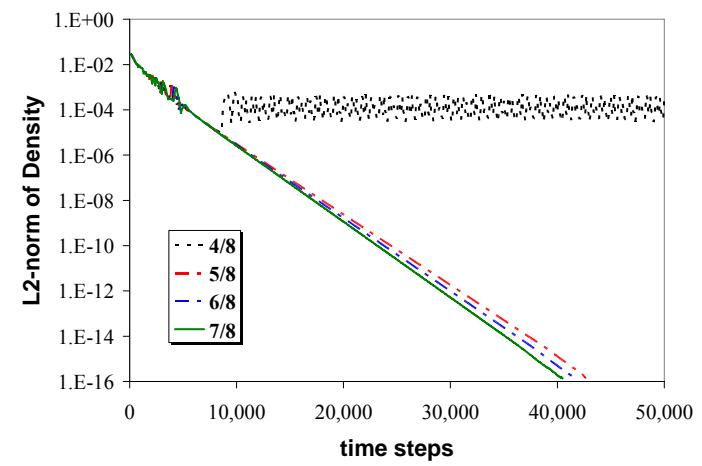

(d) $2^{\text {nd }}$-order, $4 / 8 \leq \delta \leq 7 / 8$

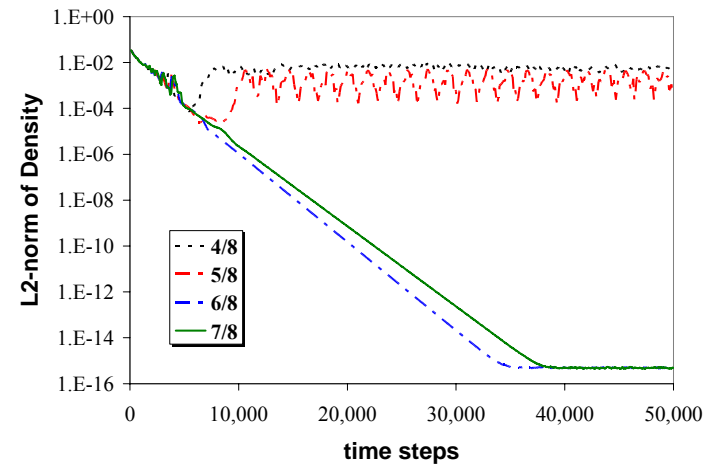

Figure 28. Residual Histories for Two Dimensional Cylinder Problem (HLLE). 